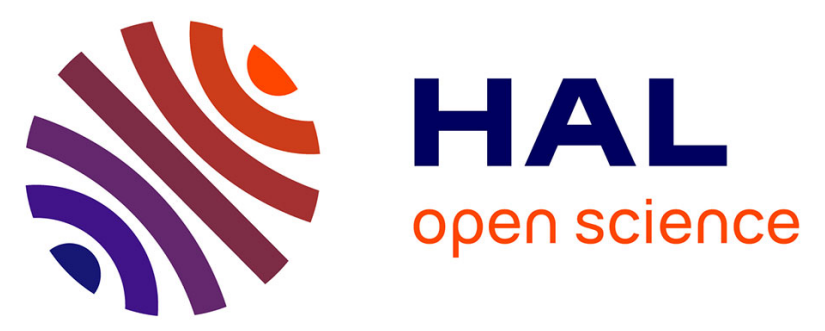

\title{
Reactivity of 1-(2-bromobenzyl)-4-halopyrazoles in intermolecular and intramolecular Pd-catalysed direct arylations
}

Mariem Brahim, Hamed Ben Ammar, Jean-François Soulé, Henri Doucet

\section{- To cite this version:}

Mariem Brahim, Hamed Ben Ammar, Jean-François Soulé, Henri Doucet. Reactivity of 1-(2bromobenzyl)-4-halopyrazoles in intermolecular and intramolecular Pd-catalysed direct arylations. Tetrahedron, 2016, 72 (29), pp.4312-4320. 10.1016/j.tet.2016.05.079 . hal-01357418

HAL Id: hal-01357418 https://hal-univ-rennes1.archives-ouvertes.fr/hal-01357418

Submitted on 14 Oct 2016

HAL is a multi-disciplinary open access archive for the deposit and dissemination of scientific research documents, whether they are published or not. The documents may come from teaching and research institutions in France or abroad, or from public or private research centers.
L'archive ouverte pluridisciplinaire HAL, est destinée au dépôt et à la diffusion de documents scientifiques de niveau recherche, publiés ou non, émanant des établissements d'enseignement et de recherche français ou étrangers, des laboratoires publics ou privés. 


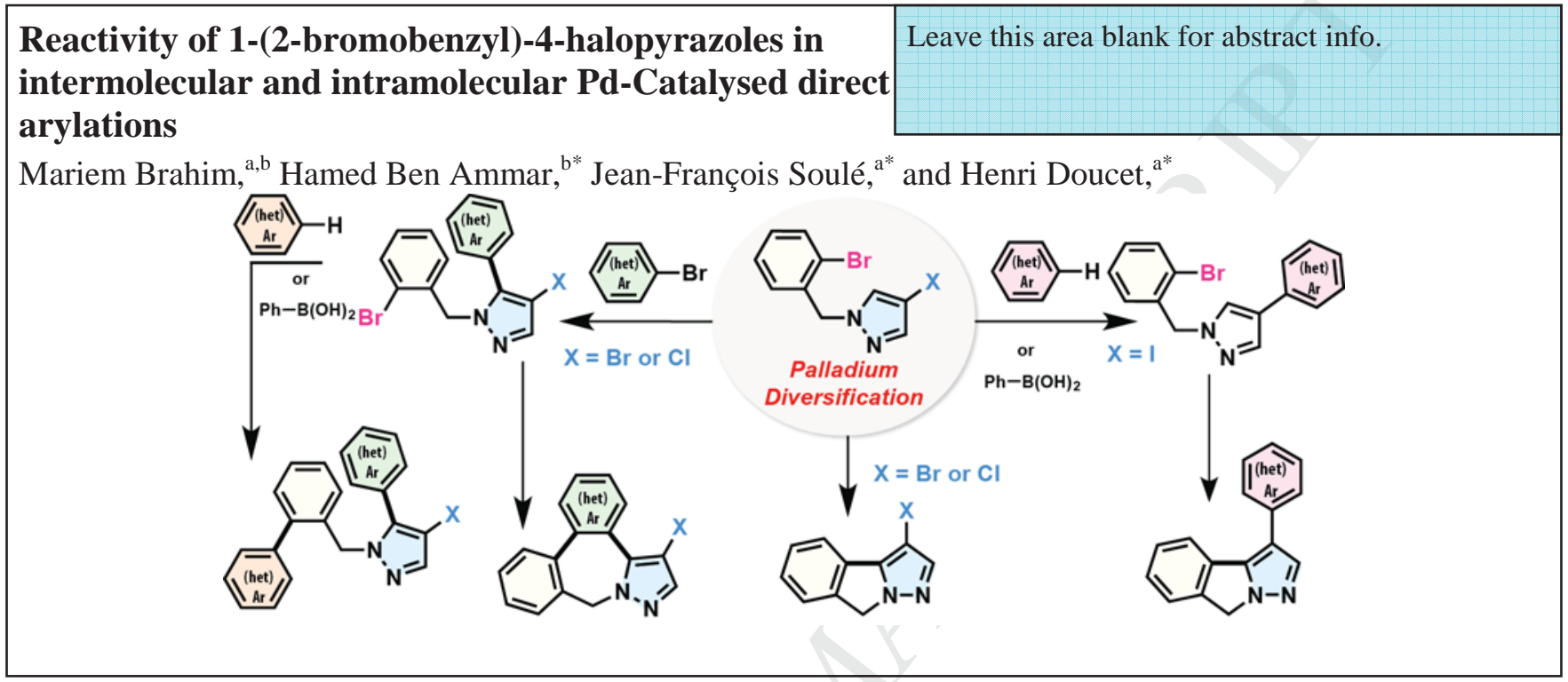


Pergamon

\title{
Reactivity of 1-(2-bromobenzyl)-4-halopyrazole $\overline{\overline{\text { in }}}$ intermolecular and intramolecular Pd-Catalysed direct arylations
}

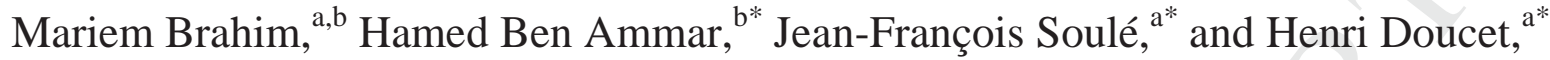

${ }^{a}$ Institut des Sciences Chimiques de Rennes, UMR 6226 CNRS-Université de Rennes

"Organométalliques: Matériaux et Catalyse", Campus de Beaulieu, 35042 Rennes, France.

${ }^{b}$ Laboratoire de Synthèse Organique Asymétrique et Catalyse Homogène, (UR 11ES56) Université de Monastir, Faculté des Sciences de Monastir, avenue de l'environnement, Monastir 5000, Tunisie. Fax: (+216) 73500 278; Tel: (+216) 73 500 275; email: henri.doucet@univ-rennes1.fr, jean-francois.soule@univ-rennes1.fr

\begin{abstract}
The reactivity of 1-(2-bromobenzyl)-4-halopyrazoles in inter- and intra-molecular Pd-catalysed direct arylation was investigated. Conditions allowing the intermolecular C5-arylations of both 1-(2-bromobenzyl)-4-chloropyrazoles and 1-(2-bromobenzyl)4-bromopyrazoles, without cleavage of the pyrazolyl and benzyl C-halo bonds, are reported. Using KOAc as the base, DMA as the solvent and $2 \mathrm{~mol} \%$ of an air stable palladium catalyst, the target C5-arylated pyrazoles were obtained in moderate to good yields with a wide variety of aryl bromides. The synthesis of 3-halopyrazolo[5,1-a]isoindoles via intramolecular Pd-catalysed direct arylation, without cleavage of the pyrazolyl C-halo bonds is also described. Moreover, sequential Pd-catalysed C5-arylations followed by intramolecular direct arylation allowed the access to dibenzo[c,e]pyrazolo[1,5-a]azepine derivatives. The reactivity of the 2-bromobenzyl moiety of C5arylated 1-(2-bromobenzyl)-4-halopyrazoles in intermolecular direct arylation or in Suzuki coupling is also described. (C) 2016 Elsevier Science. All rights reserved
\end{abstract}

\section{Introduction}

Pyrazole derivatives including those bearing alkyl-, aryl- or halo-substituents are important structures due to their biological properties (Fig 1). For example, Deracoxib and Mavacoxib are non-steroidal anti-inflammatory drugs used in veterinary medicine to treat osteoarthritis in dogs. Afuresertib shows activity in multiple myeloma. Meclinertant is a drug which acts as a selective, nonpeptide antagonist at the neurotensin receptor $\mathrm{NTS}_{1}$. Nelotanserin exhibits properties in neurological diseases. Due to these multiple uses, the discovery of simpler routes for accesses to a variety of alkyl-, aryl- or halo-substituted pyrazoles remains an important research topic in organic synthesis.

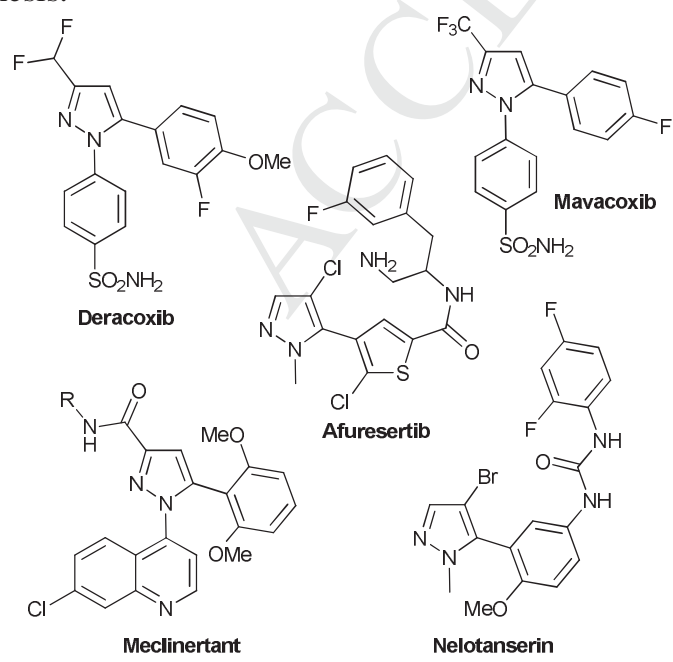

Figure 1. Examples of bioactive 5-(hetero)arylpyrazoles
Stille, Suzuki or Negishi Pd-catalysed coupling reactions represent some of the most efficient methods to prepare (hetero)arylpyrazoles; however, such reactions require the previous preparation of an organometallic derivative. ${ }^{1}$ In 1985, Ohta et al. reported the Pd-catalysed direct arylation of heteroaromatics via a $\mathrm{C}-\mathrm{H}$ bond activation using aryl halides as arylating agents. ${ }^{2}$ Since these results, this methodology proved to be a very powerful tool for a simpler and greener access to a very wide variety of arylated heterocycles, as it avoids the preparation of an organometallic derivative and as the major by-products of the reaction are a base associated to $\mathrm{HX}^{3}$ Several examples of Pd-catalysed direct arylations of pyrazoles using aryl halides as coupling partners have been reported in recent years. ${ }^{4}$ However, to our knowledge, only a few examples of such arylations dealing with the reactivity of $N$-benzylpyrazoles have been described. ${ }^{5,6}$ In 2014, the C5arylation of a 4-nitro- $N$-benzylpyrazole has been reported by Langer et al. (Scheme 1, top). ${ }^{5}$ The introduction of a nitro-substituent at pyrazolyl C4-position allowed to control the regioselectivity of the reaction. The intramolecular Pd-catalysed direct arylation of 1-(2bromobenzyl)-pyrazoles for the synthesis of pyrazolo[5,1a]isoindoles has been reported by Heo et al. (Scheme 1, middle). ${ }^{6}$ They observed that the use of $10 \mathrm{~mol} \%$ of $\mathrm{Pd}(\mathrm{OAc})_{2}$ catalyst promotes this intramolecular reaction. So far, only a few examples of Pd-catalysed direct C5arylations of 4-halo-substituted pyrazoles have been reported. $^{7}$ 


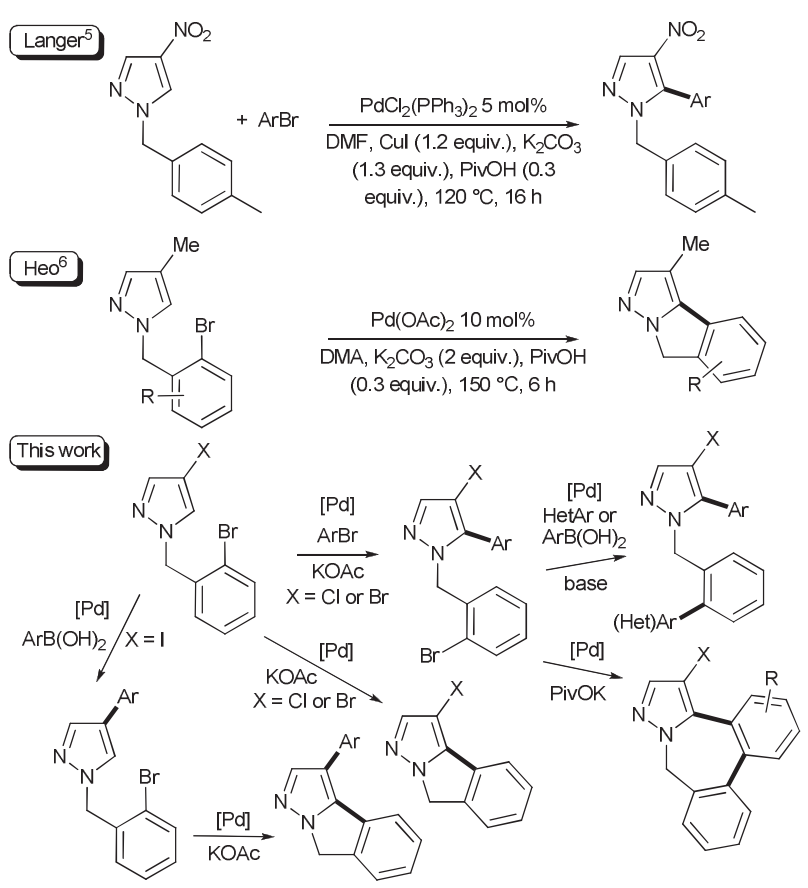

Scheme 1.

To our knowledge, Pd-catalysed direct inter- or intramolecular arylations using 1-(2-bromobenzyl)-4halopyrazoles have not been described. Therefore, their reactivity needed to be investigated. Here, we wish to report conditions allowing the sequential C5-arylation of such 1-(2-bromobenzyl)-4-halopyrazoles, without cleavage of the pyrazolyl and benzyl C-halo bonds, followed by i) heteroarylation of the benzyl unit via an intermolecular Pdcatalysed reaction, ii) arylation of the benzyl unit via Suzuki coupling, iii) intramolecular Pd-catalysed direct arylation for access to dibenzo[c,e]pyrazolo[1,5-a]azepines. The reactivity of 1-(bromobenzyl)-4-halopyrazoles in intramolecular Pd-catalysed direct arylation is also reported.

\section{Results and discussion}

The 1-(2-bromobenzyl)-4-halopyrazoles $\mathbf{1}$ and $\mathbf{3}$ were prepared by reaction of 4-chloropyrazole or 4-iodopyrazole with 2-bromobenzyl bromide (Scheme 2, top). Compound 2 was prepared by reaction of 1-(2-bromobenzyl)-pyrazole with $N$-bromosuccinimide (Scheme 2, bottom).
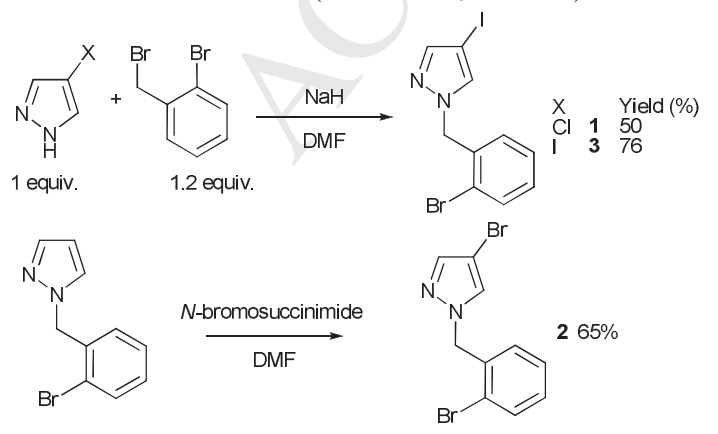

Scheme 2. Synthesis of the 1-(2-bromobenzyl)-4halopyrazoles 1-3.

Then, the reactivity of $\mathbf{1 - 3}$ in both inter- and intramolecular Pd-catalysed direct arylations was investigated. Firstly, we studied the intermolecular reaction of 1-3 with aryl bromides (Schemes 3-5). Based on our previous results, DMA was chosen as the solvent and KOAc as the base. ${ }^{8}$ The reactions were conducted at $150{ }^{\circ} \mathrm{C}$ under argon using $\mathrm{PdCl}\left(\mathrm{C}_{3} \mathrm{H}_{5}\right)(\mathrm{dppb})$ catalyst. The reaction of 1 equiv. of 1-(2-bromobenzyl)-4-chloropyrazole 1 with 1.5 equiv. of 4-bromobenzonitrile affords the desired C5-arylated pyrazoles 4 in 71\% yield (Scheme 3). Both $\mathrm{Pd}(\mathrm{OAc})_{2}$ and $\mathrm{PdCl}_{2}$ catalysts (2 $\mathrm{mol} \%$ ) were found to be completely ineffective for this reaction. It should be mentioned that no intramolecular direct arylation of $\mathbf{1}$ was observed and that both $\mathrm{C}-\mathrm{Cl}$ and $\mathrm{C}-\mathrm{Br}$ bonds of $\mathbf{1}$ remained untouched. A similar reactivity was observed in the presence of 4bromonitrobenzene, 4-bromobenzaldehyde, 4-bromo-1nitro-2-(trifluoromethyl)benzene, 3-bromoacetophenone or 3,5-bis(trifluoromethyl)bromobenzene with the formation of compounds 5-9 in 62-75\% yields. A very high yield of $90 \%$ in 10 was obtained for the coupling of $\mathbf{1}$ with 2bromobenzonitrile; whereas, the use of 2bromobenzaldehyde gave $\mathbf{1 1}$ in only 43\%, due to the formation of side products. The reaction proceeds very smoothly with 3-bromopyridine, 3-bromoquinoline and also 5-bromopyrimidine affording the products 12-14 in $76-88 \%$ yields.
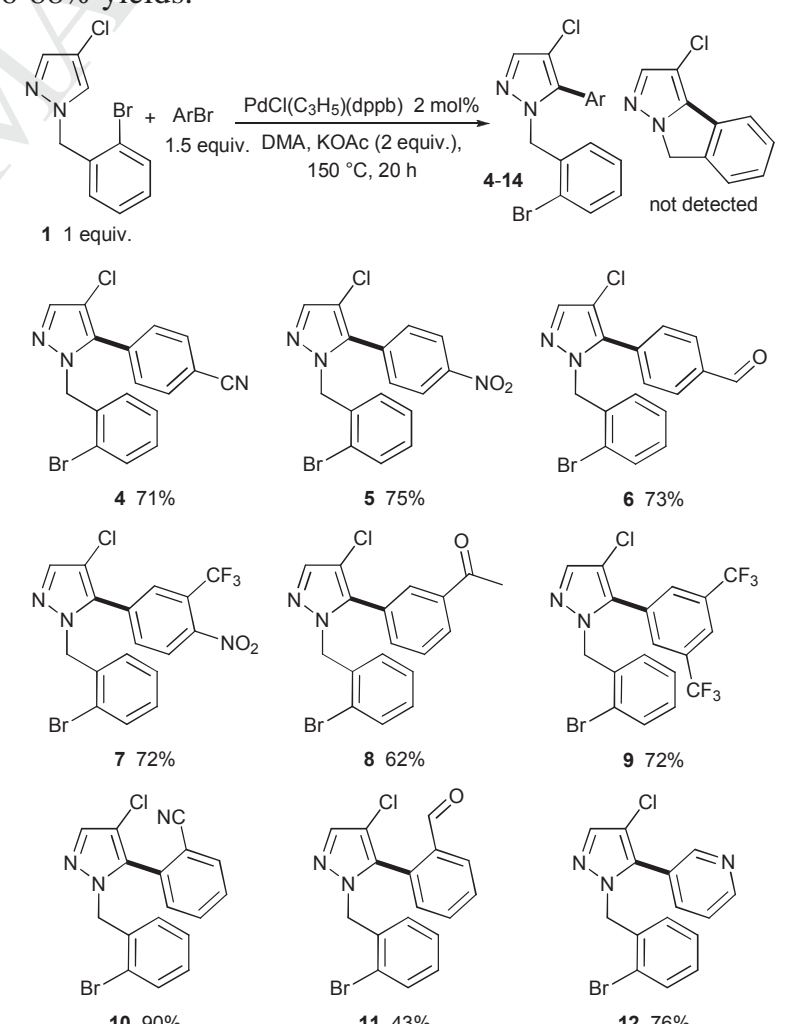

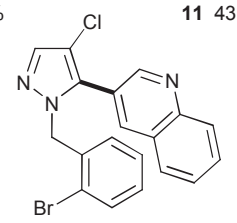

$1386 \%$

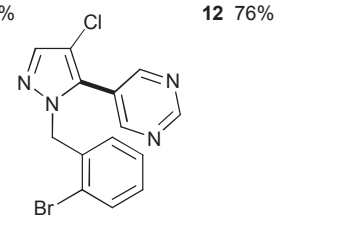


Scheme 3. Reactivity of 1-(2-bromobenzyl)-4chloropyrazole 1 with (hetero)aryl bromides.

Then, the reactivity of 1-(2-bromobenzyl)-4-bromopyrazole 2 in intermolecular C5-arylation was explored using 2 mol\% $\mathrm{PdCl}\left(\mathrm{C}_{3} \mathrm{H}_{5}\right)(\mathrm{dppb})$ catalyst, KOAc as base in DMA at $150{ }^{\circ} \mathrm{C}$ (Scheme 4). From a set of electron-deficient aryl bromides, the C5-arylated pyrazoles 15-25 were obtained in moderate to high yields. The reaction tolerates para-, meta- and ortho-substituted aryl bromides and also heteroaryl bromides. Again, no formation of cyclized product via intramolecular Pd-catalysed direct arylation of 2 was observed. Moreover, although a quite elevated temperature was employed for these couplings, no cleavage of the pyrazolyl C-Br bond was detected. The reactivity of 2 with 4-bromonitrobenzene or 4-bromobenzonitrile using $2 \mathrm{~mol} \% \mathrm{Pd}(\mathrm{OAc})_{2}$ or $\mathrm{PdCl}_{2}$ catalysts was also investigated. However, 15 and $\mathbf{1 6}$ were obtained in lower yields than in the presence of $\operatorname{PdCl}\left(\mathrm{C}_{3} \mathrm{H}_{5}\right)(\mathrm{dppb})$ catalyst.
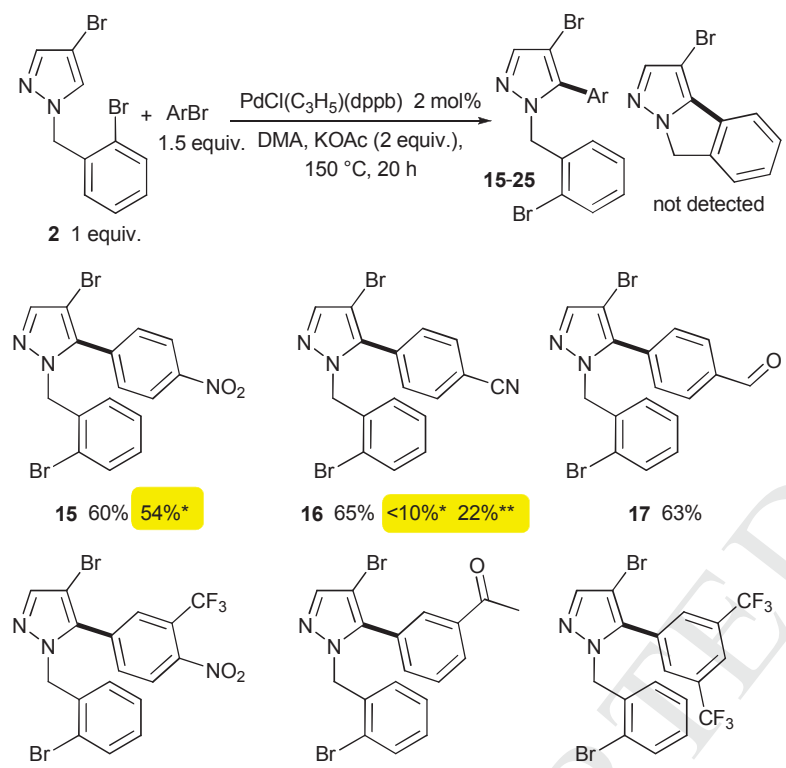

$1665 \%<10 \%{ }^{*} 22 \%$ **

$1763 \%$
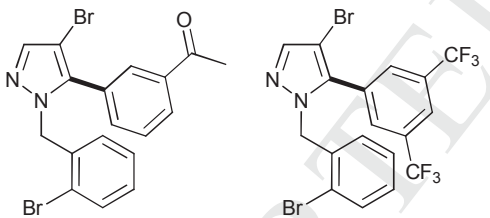

$1944 \%$

$2081 \%$

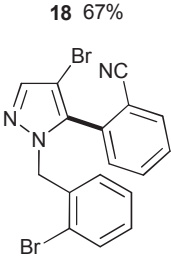

$2177 \%$

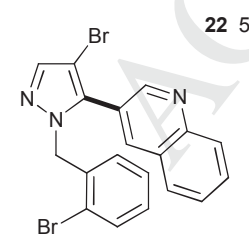

$2456 \%$

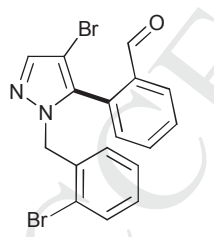

$55 \%$

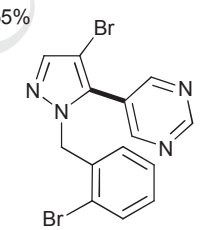

$2574 \%$

* $2 \mathrm{~mol} \% \mathrm{Pd}(\mathrm{OAc})_{2}$ as catalyst * $2 \mathrm{~mol} \% \mathrm{PdCl}_{2}$ as catalyst

Scheme 4. Reactivity of 1-(2-bromobenzyl)-4bromopyrazole 2 with (hetero)aryl bromides.
If both 4-chloro- and 4-bromo-subtituents on $\mathrm{N}$-(2bromobenzyl)pyrazoles are tolerated in Pd-catalysed intermolecular direct C5-arylation; on the other hand, a mixture of the 4-iodo-substituted pyrazole $\mathbf{3}$ and 4bromobenzonitrile or 4-bromonitrobenzene failed to afford the desired C5-arylated pyrazoles (Scheme 5). At $150^{\circ} \mathrm{C}$ or $120^{\circ} \mathrm{C}$, a large amount of de-iodination side-product was observed via GC/MS analysis; whereas, at $80^{\circ} \mathrm{C}, 3$ was recovered.
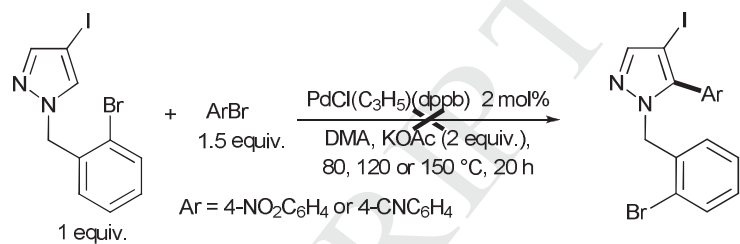

Scheme 5. Reactivity of 1-(2-bromobenzyl)-4-iodopyrazole $\mathbf{3}$ with aryl bromides.

The reactivity of the 2-bromobenzyl moiety in intermolecular Pd-catalysed arylation with an heteroarene was then studied (Scheme 6). The reaction of 1-(2bromobenzyl)-4-chloro-5-(4-nitrophenyl)-pyrazole 4 and 2ethyl-4-methylthiazole in the presence of 2 mol\% $\mathrm{PdCl}\left(\mathrm{C}_{3} \mathrm{H}_{5}\right)(\mathrm{dppb})$ gave 26 in $89 \%$ yield. A similar result was observed with 2-methylthiophene as reaction partner, and 27 was produced in $86 \%$ yield. The 1-(2bromobenzyl)-pyrazole $\mathbf{1 0}$ bearing a benzonitrile at C5position also reacted nicely with 2-ethyl-4-methylthiazole affording 28 in $90 \%$ yield. A slightly lower yield of $83 \%$ in 29 was obtained from a pyrazole substituted at C5 by a pyridine and 2-n-butylfuran. In all cases, no intramolecular reaction with activation of a $\mathrm{C}-\mathrm{H}$ bond of the nitrophenyl, benzonitrile or pyridyl moieties was observed, and again the pyrazolyl C-Cl bond remained untouched.
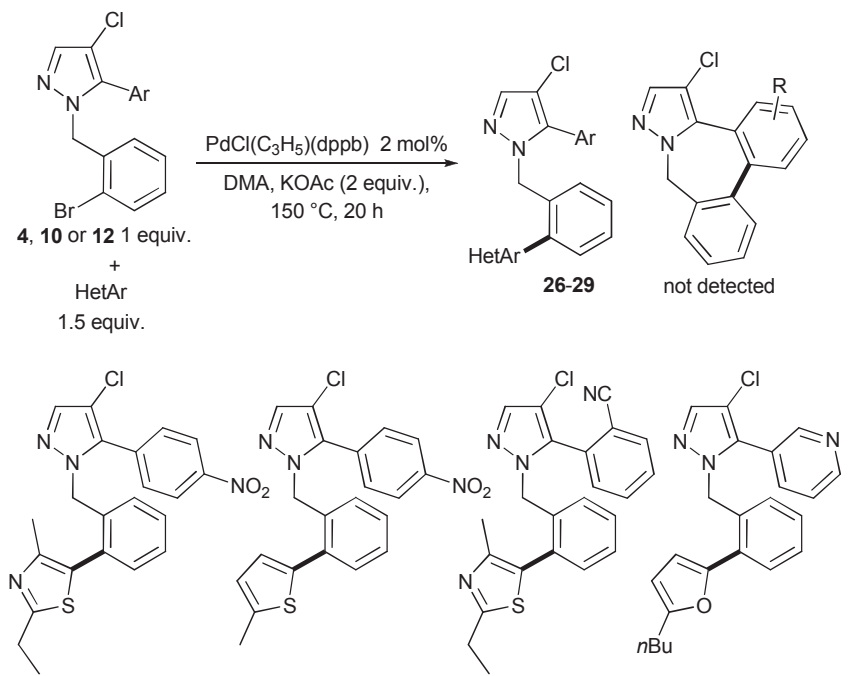

$2689 \%$

$2786 \%$

$2890 \%$

$2983 \%$

Scheme 6. Reactivity of C5-arylated 1-(2-bromobenzyl)-4chloropyrazoles 4, 10 and 12 with heteroarenes.

The reactivity of the two C5-arylated 1-(2-bromobenzyl)-4chloropyrazoles $\mathbf{1 0}$ and $\mathbf{2 4}$ in Suzuki type coupling was 
also evaluated (Scheme 7). From 10 and phenylboronic acid in the presence of $2 \mathrm{~mol} \% \mathrm{Pd}(\mathrm{OAc})_{2}$, the desired product 30 was obtained in $88 \%$ yield. No cleavage of the pyrazolyl C-Cl bonds was observed. Similar results were obtained from 16 and 24, as the desired compounds 31 and 32 were isolated in $77 \%$ and $84 \%$ yield, respectively. These results reveal that in $\mathbf{1 6}$ and $\mathbf{2 4}$, the benzyl C-Br bond is more reactive than the pyrazolyl $\mathrm{C}-\mathrm{Br}$ bond.

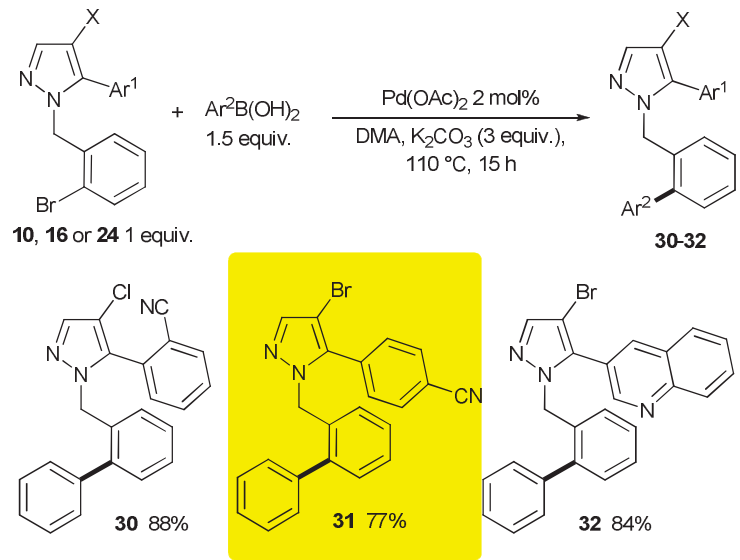

Scheme 7. Reactivity of C5-arylated 1-(2-bromobenzyl)-4halopyrazoles $\mathbf{1 0}$ and $\mathbf{2 4}$ with arylboronic acids.

The intramolecular Pd-catalysed direct arylation using 1-3 for the formation of 5-membered rings was also attempted (Scheme 8). 4-Chloro-substituted pyrazole 1 reacts nicely in the presence of $2 \mathrm{~mol} \% \operatorname{PdCl}\left(\mathrm{C}_{3} \mathrm{H}_{5}\right)(\mathrm{dppb})$ catalyst affording 32 in $88 \%$ yield. In order to determine the reactivity of $\mathbf{1}$ in intermolecular $v$ s intramolecular direct arylation, the reaction outcome of a mixture of $\mathbf{1}$ and 2ethyl-4-methylthiazole in the presence of $2 \mathrm{~mol} \%$ $\operatorname{PdCl}\left(\mathrm{C}_{3} \mathrm{H}_{5}\right)(\mathrm{dppb})$ catalyst was studied. An almost exclusive formation of the intramolecular reaction product 33 was observed. A good yield in desired cyclised product 34 was obtained from the 4-bromo-substituted pyrazole 2. Moreover, the pyrazoly $\mathrm{C}-\mathrm{Br}$ bond remained untouched. Again, the oxidative addition of the benzyl $\mathrm{C}-\mathrm{Br}$ bond to palladium appears to be faster than the pyrazoly $\mathrm{C}-\mathrm{Br}$ bond.
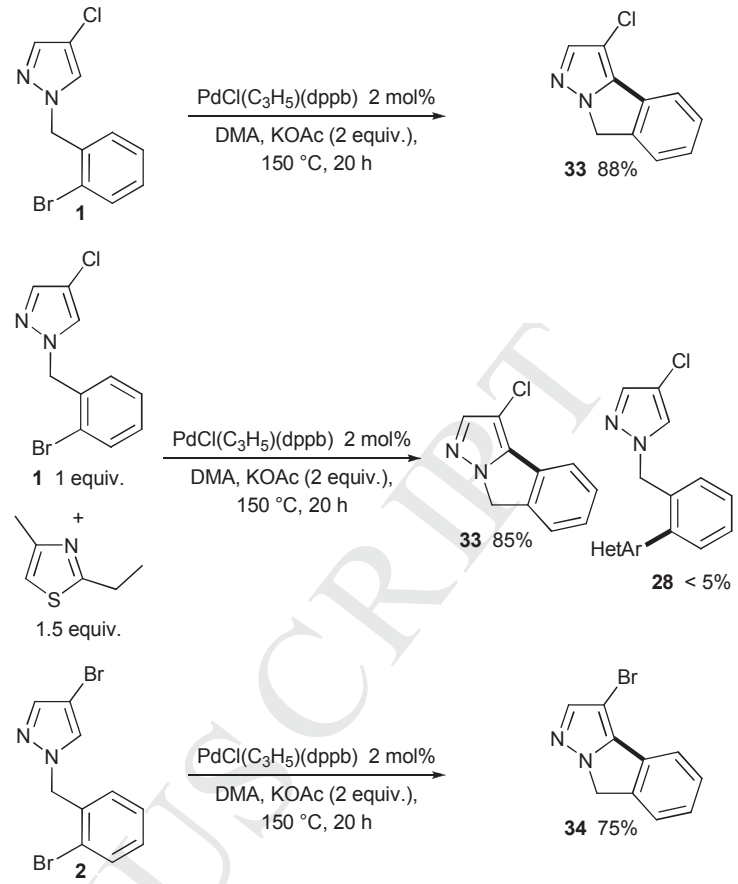

Scheme 8. Reactivity of 1-(2-bromobenzyl)-4halopyrazoles $\mathbf{1}$ and $\mathbf{2}$ in Pd-catalysed intramolecular arylation.

On the other hand, from the 4-iodo-substituted pyrazole 3, under the same reaction conditions, no formation of the desired cyclised product 35 was observed in GC/MS analysis of the crude mixture (Scheme 9). As in 3, pyrazolyl C-I bond should exhibit a higher reactivity than the benzyl $\mathrm{C}-\mathrm{Br}$ bond, it was employed to introduce (hetero)aryls substituents at C4. The reaction of $\mathbf{3}$ with phenylboronic acid or 2-thienylboronic acid in the presence of $1 \mathrm{~mol} \% \mathrm{PdCl}\left(\mathrm{C}_{3} \mathrm{H}_{5}\right)(\mathrm{dppb})$ affords 36 and 37 in 54\% and $62 \%$ yields, respectively. Then, intramolecular Pdcatalysed arylation of 36 and 37 gave the 3(hetero)arylpyrazolo[5,1-a]isoindoles $\mathbf{3 8}$ and $\mathbf{3 9}$ in almost quantitative yields. It should be mentioned that no intermolecular Pd-catalysed arylation involving a C-H bond of the thienyl moiety of $\mathbf{3 6}$ was observed.
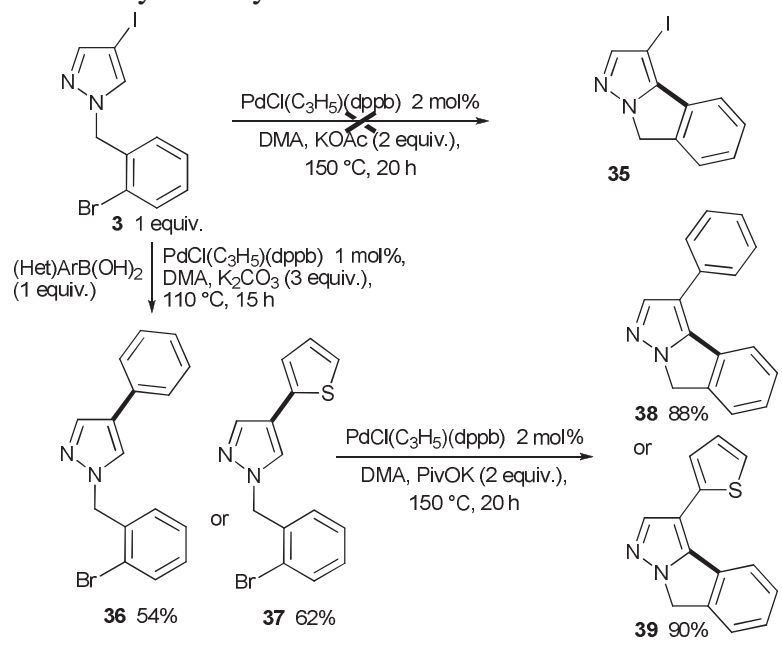
Scheme 9. Reactivity of 1-(2-bromobenzyl)-4-iodopyrazole 3 in Pd-catalysed intramolecular arylation.

Finally, the intramolecular Pd-catalysed direct arylation of the two C5-arylated 1-(2-bromobenzyl)-4-halopyrazoles $\mathbf{1 0}$ and 21, in order to prepare dibenzo[c,e]pyrazolo[1,5a]azepine derivatives, via the formation of a 7-membered ring, was attempted (Scheme 10). It should be mentioned that, to our knowledge, such structures have not yet been described, revealing that their access is quite challenging. The reaction of $\mathbf{1 0}$ in the presence of $2 \mathrm{~mol} \%$ $\operatorname{PdCl}\left(\mathrm{C}_{3} \mathrm{H}_{5}\right)(\mathrm{dppb})$ catalyst and PivOK as base led to the target product $\mathbf{4 0}$ in $28 \%$ yield. For this reaction the use of KOAc as base was ineffective. A similar influence of the nature of the base had been previously observed in the Pdcatalysed intramolecular direct arylation of imidazole derivatives. $^{9} \quad$ A slightly higher yield of $35 \%$ in $\mathbf{4 1}$ was obtained from the 4-bromosubstituted pyrazole derivative 21. Even if the yields of these two reactions are moderate, this is the first method allowing the preparation of this type of dibenzopyrazoloazepine derivatives.
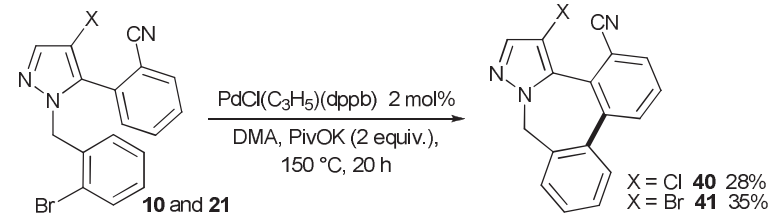

Scheme 10. Reactivity of 1-(2-bromobenzyl)-4halopyrazoles $\mathbf{1 0}$ and $\mathbf{2 1}$ in Pd-catalysed intramolecular arylation.

\section{Conclusion}

We established that under appropriate reaction conditions, the intermolecular palladium-catalysed C5-arylation of 1(2-bromobenzyl)-4-chloropyrazole 1 or 1-(2-bromobenzyl)4-bromopyrazole 2 proceeds nicely, without cleavage of both pyrazolyl and benzyl C-halo bonds. A wide variety of aryl bromides was successfully employed. On the other hand, with 1-(2-bromobenzyl)-4-iodopyrazole 3, degradation products were formed. The synthesis of 3halopyrazolo[5,1-a]isoindoles (with halo $=\mathrm{Br}$ or $\mathrm{Cl}$ ) via intramolecular Pd-catalysed direct arylation, without cleavage of the pyrazolyl C-halo bonds, was also found to proceed in high yields. The sequential Pd-catalysed C5arylation followed by intramolecular direct arylation allowed the preparation of dibenzo[c,e]pyrazolo[1,5a]azepine derivatives. The intermolecular direct arylation or Suzuki coupling of the 2-bromobenzyl moiety of C5arylated 1-(2-bromobenzyl)-4-halopyrazoles is also reported. These results demonstrate that the use of an appropriate C4-halo substituent associated to a 2bromobenzyl moiety on pyrazoles allows to prepare a wide variety of pyrazoles derivatives via successive inter- or intra-molecular Pd-catalysed couplings.

\section{Acknowledgments}

We thank the Centre National de la Recherche Scientifique, "Rennes Metropole”, “UTIQUE” and Scientific Ministry of
Higher Education Research of Tunisia for providing financial support.

\section{Experimental section}

General Remarks: All catalytic reactions were carried out under argon atmosphere with standard Schlenk techniques. DMA ( $N, N$-dimethylacetamide) (99\%) was purchased from Acros. KOAc (99\%) was purchased from Alfa Aesar. These compounds were not purified before use. The 4halopyrazoles were prepared from NCS, NBS or $\mathrm{I}_{2}$ and pyrazoles according to reported procedures. ${ }^{10}{ }^{1} \mathrm{H}$ NMR spectra were recorded on Bruker GPX (400 MHz) spectrometer. Chemical shifts $(\delta)$ were reported in parts per million relative to residual chloroform (7.26 ppm for ${ }^{1} \mathrm{H}$; 77.0 ppm for ${ }^{13} \mathrm{C}$ ), constants were reported in Hertz. ${ }^{1} \mathrm{H}$ NMR assignment abbreviations were the following: singlet $(\mathrm{s})$, doublet (d), triplet ( $\mathrm{t}$ ), quartet $(\mathrm{q})$, doublet of doublets (dd), doublet of triplets (dt), and multiplet (m). ${ }^{13} \mathrm{C}$ NMR spectra were recorded at $100 \mathrm{MHz}$ on the same spectrometer and reported in ppm. All reagents were weighed and handled in air.

\section{1-(2-Bromobenzyl)-4-chloropyrazole (1) 4-}

Chloropyrazole (1.02 g, $10 \mathrm{mmol})$, 2-bromobenzylbromide (3.00 g, $12 \mathrm{mmol})$ and $\mathrm{NaH}(0.24 \mathrm{~g}, 10 \mathrm{mmol})$ in DMF (50 $\mathrm{mL}$ ) were stirred at $0^{\circ} \mathrm{C}$ during $14 \mathrm{~h}$. The mixture was poured on ice, extracted with ethyl acetate, dried over $\mathrm{MgSO}_{4}$ and filtered. After concentration in vacuum, the residue was purified by flash-chromatography on silica gel to afford 1 in 50\% (1.36 g) yield. ${ }^{1} \mathrm{H}$ NMR (400 MHz, $\left.\mathrm{CDCl}_{3}\right) \delta 7.56(\mathrm{~d}, J=8.2 \mathrm{~Hz}, 1 \mathrm{H}), 7.47(\mathrm{~s}, 1 \mathrm{H}), 7.42$ (s, 1H), 7.27 (t, $J=7.8 \mathrm{~Hz}, 1 \mathrm{H}), 7.17$ (t, $J=7.8 \mathrm{~Hz}, 1 \mathrm{H}), 7.01$ $(\mathrm{d}, J=8.2 \mathrm{~Hz}, 1 \mathrm{H}), 5.34(\mathrm{~s}, 2 \mathrm{H}) .{ }^{13} \mathrm{C} \mathrm{NMR}(100 \mathrm{MHz}$, $\left.\mathrm{CDCl}_{3}\right) \delta 138.2,135.3,133.1,130.0,129.9,128.0,127.8$, 123.2, 110.4, 56.4 .

4-Bromo-1-(2-bromobenzyl)-pyrazole (2) 1-(2bromobenzyl)-pyrazole (2.37 g, $10 \mathrm{mmol})$ and $\mathrm{N}$ bromosuccinimide $(2.14,12 \mathrm{mmol})$ in $\mathrm{MeCN}(50 \mathrm{~mL})$ were stirred at $25^{\circ} \mathrm{C}$ during $3 \mathrm{~h}$. The mixture was poured on ice, extracted with ethyl acetate, dried over $\mathrm{MgSO}_{4}$ and filtered. After concentration in vacuum, the residue was purified by flash-chromatography on silica gel to afford 2 in 65\% (2.05 g) yield. ${ }^{1} \mathrm{H}$ NMR (400 MHz, $\left.\mathrm{CDCl}_{3}\right) \delta 7.60$ (d, $J=8.2$ Hz, 1H), 7.51 (s, 1H), 7.47 (s, 1H), 7.29 (t, $J=7.8 \mathrm{~Hz}, 1 \mathrm{H}$ ), 7.19 (t, $J=7.8 \mathrm{~Hz}, 1 \mathrm{H}), 7.04$ (d, $J=8.2 \mathrm{~Hz}, 1 \mathrm{H}), 5.39$ (s, 2H). ${ }^{13} \mathrm{C} \mathrm{NMR}\left(100 \mathrm{MHz}, \mathrm{CDCl}_{3}\right) \delta 140.6,135.5,133.3$, 130.3, 130.2 (x2), 128.3, 123.5, 93.8, 56.6.

1-(2-Bromobenzyl)-4-iodopyrazole (3) 4-Iodopyrazole (1.94 g, $10 \mathrm{mmol}$ ), 2-bromobenzylbromide (3.00 g, 12 $\mathrm{mmol})$ and $\mathrm{NaH}(0.24 \mathrm{~g}, 10 \mathrm{mmol})$ in DMF (50 mL) were stirred at $0^{\circ} \mathrm{C}$ during $14 \mathrm{~h}$. The mixture was poured on ice, extracted with ethyl acetate, dried over $\mathrm{MgSO}_{4}$ and filtered. After concentration in vacuum, the residue was purified by flash-chromatography on silica gel to afford 3 in 76\% (2.76 g) yield. ${ }^{1} \mathrm{H}$ NMR (400 MHz, $\left.\mathrm{CDCl}_{3}\right) \delta 7.59$ (d, $J=8.2$ 
Hz, 1H), 7.57 (s, 1H), 7.50 (s, 1H), 7.27 (t, $J=7.8 \mathrm{~Hz}, 1 \mathrm{H}$ ), $7.21(\mathrm{t}, J=7.8 \mathrm{~Hz}, 1 \mathrm{H}), 7.04(\mathrm{~d}, J=8.2 \mathrm{~Hz}, 1 \mathrm{H}), 5.42$ (s, 2H). $\left.{ }^{13} \mathrm{C} \mathrm{NMR} \mathrm{(100} \mathrm{MHz,} \mathrm{CDCl}_{3}\right) \delta 145.1$ 135.6, 134.5, 133.3, 130.2, 128.2, 123.5, 56.4.

Preparation of the $\operatorname{PdCl}\left(\mathrm{C}_{3} \mathbf{H}_{5}\right)(\mathbf{d p p b})$ catalyst: $^{11} \mathrm{An}$ oven-dried $40 \mathrm{~mL}$ Schlenk tube equipped with a magnetic stirring bar under argon atmosphere, was charged with $\left[\mathrm{Pd}\left(\mathrm{C}_{3} \mathrm{H}_{5}\right) \mathrm{Cl}\right]_{2}(182 \mathrm{mg}, 0.5 \mathrm{mmol})$ and dppb (426 mg, 1 $\mathrm{mmol}) .10 \mathrm{~mL}$ of anhydrous dichloromethane were added, then, the solution was stirred at room temperature for twenty minutes. The solvent was removed in vacuum. The yellow powder was used without purification. ${ }^{31} \mathrm{P}$ NMR $\left(81 \mathrm{MHz}, \mathrm{CDCl}_{3}\right) \delta=19.3$ (s).

General procedure for the preparation of 4-25: The reaction of the 4-halopyrazole derivative (1 mmol), aryl bromide (1.5 mmol), and KOAc (0.196 g, $2 \mathrm{mmol})$ at $150^{\circ} \mathrm{C}$ during $20 \mathrm{~h}$ in DMA (4 mL) in the presence of $\mathrm{PdCl}\left(\mathrm{C}_{3} \mathrm{H}_{5}\right)(\mathrm{dppb})(12.2 \mathrm{mg}, 0.02 \mathrm{mmol})$ under argon affords the coupling product after evaporation of the solvent and purification on silica gel.

4-(1-(2-Bromobenzyl)-4-chloropyrazol-5-yl)benzonitrile (4) From 1-(2-bromobenzyl)-4-chloropyrazole 1 (0.271 g, $1 \mathrm{mmol})$ and 4-bromobenzonitrile (0.273 g, $1.5 \mathrm{mmol})$ product 4 was obtained in $71 \%$ (0.264 g) yield.

${ }^{1} \mathrm{H}$ NMR (400 MHz, $\left.\mathrm{CDCl}_{3}\right) \delta 7.72(\mathrm{~d}, J=8.4 \mathrm{~Hz}, 2 \mathrm{H})$, 7.66 (s, $1 \mathrm{H}), 7.52$ (d, $J=8.2 \mathrm{~Hz}, 1 \mathrm{H}), 7.42$ (d, $J=8.4 \mathrm{~Hz}$, 2H), 7.26 (t, $J=7.8 \mathrm{~Hz}, 1 \mathrm{H}$ ), 7.16 (t, $J=7.8 \mathrm{~Hz}, 1 \mathrm{H}), 6.80$ $(\mathrm{d}, J=8.2 \mathrm{~Hz}, 1 \mathrm{H}), 5.34(\mathrm{~s}, 2 \mathrm{H}) .{ }^{13} \mathrm{C}$ NMR $(100 \mathrm{MHz}$, $\left.\mathrm{CDCl}_{3}\right) \delta 138.6,138.5,135.8,133.0,132.7,132.2,130.3$, 129.7, 128.4, 128.1, 122.0, 118.3, 113.3, 110.3, 54.6. $\mathrm{C}_{17} \mathrm{H}_{11} \mathrm{BrClN}_{3}$ (372.65): Calcd C 54.79, H 2.98; Found C 54.70, H 3.10.

1-(2-Bromobenzyl)-4-chloro-5-(4-nitrophenyl)-pyrazole (5) From 1-(2-bromobenzyl)-4-chloropyrazole 1 (0.271 g, $1 \mathrm{mmol}$ ) and 4-bromonitrobenzene (0.303 g, $1.5 \mathrm{mmol})$ product 5 was obtained in $75 \%$ (0.294 g) yield. ${ }^{1} \mathrm{H}$ NMR $\left(400 \mathrm{MHz}, \mathrm{CDCl}_{3}\right) \delta 8.30(\mathrm{~d}, J=8.4 \mathrm{~Hz}, 2 \mathrm{H}), 7.69(\mathrm{~s}, 1 \mathrm{H})$, 7.54 (d, $J=8.2 \mathrm{~Hz}, 1 \mathrm{H}), 7.50$ (d, $J=8.4 \mathrm{~Hz}, 2 \mathrm{H}), 7.28$ (t, $J$ $=7.8 \mathrm{~Hz}, 1 \mathrm{H}), 7.19(\mathrm{t}, J=7.8 \mathrm{~Hz}, 1 \mathrm{H}), 6.83(\mathrm{~d}, J=8.2 \mathrm{~Hz}$, $1 \mathrm{H}), 5.37$ (s, $2 \mathrm{H}) .{ }^{13} \mathrm{C} \mathrm{NMR}\left(100 \mathrm{MHz}, \mathrm{CDCl}_{3}\right) \delta 148.6$, 139.0, 138.6, 136.1, 134.4, 133.4, 130.9, 130.1, 128.7, 128.4, 124.5, 122.3, 110.9, 55.0. $\mathrm{C}_{16} \mathrm{H}_{11} \mathrm{BrClN}_{3} \mathrm{O}_{2}$ (392.63): Calcd C 48.94, H 2.82; Found C 48.78, H 2.74.

\section{4-(1-(2-Bromobenzyl)-4-chloropyrazol-5-}

yl)benzaldehyde (6) From 1-(2-bromobenzyl)-4chloropyrazole $1 \quad(0.271 \mathrm{~g}, \quad 1 \mathrm{mmol})$ and 4bromobenzaldehyde $(0.278 \mathrm{~g}, 1.5 \mathrm{mmol})$ product 6 was obtained in 73\% (0.273 g) yield. ${ }^{1} \mathrm{H}$ NMR (400 MHz, $\left.\mathrm{CDCl}_{3}\right) \delta 10.06(\mathrm{~s}, 1 \mathrm{H}), 7.94(\mathrm{~d}, J=8.4 \mathrm{~Hz}, 2 \mathrm{H}), 7.67$ (s, $1 \mathrm{H}), 7.52$ (d, $J=8.2 \mathrm{~Hz}, 1 \mathrm{H}), 7.49$ (d, $J=8.4 \mathrm{~Hz}, 2 \mathrm{H}), 7.26$ (t, $J=7.8 \mathrm{~Hz}, 1 \mathrm{H}), 7.16$ (t, $J=7.8 \mathrm{~Hz}, 1 \mathrm{H}), 6.80$ (d, $J=8.2$ $\mathrm{Hz}, 1 \mathrm{H}), 5.36(\mathrm{~s}, 2 \mathrm{H}),{ }^{13} \mathrm{C} \operatorname{NMR}\left(100 \mathrm{MHz} \mathrm{CDCl}_{3}\right) \delta$
191.6, 139.2, 138.6, 136.7, 136.0, 133.5, 133.0, 130.3, 130.1, 129.6, 128.4, 128.0, 122.0, 110.2, 54.6. $\mathrm{C}_{17} \mathrm{H}_{12} \mathrm{BrClN}_{2} \mathrm{O}$ (375.65): Calcd C 54.35, H 3.22; Found C 54.40, H 3.04.

\section{1-(2-Bromobenzyl)-4-chloro-5-(4-nitro-3-}

(trifluoromethyl)phenyl)-pyrazole (7) From 1-(2bromobenzyl)-4-chloropyrazole 1 (0.271 g, $1 \mathrm{mmol})$ and 4bromo-1-nitro-2-(trifluoromethyl)benzene (0.405 g, 1.5 mmol) product 7 was obtained in $72 \%(0.331 \mathrm{~g})$ yield. ${ }^{1} \mathrm{H}$ NMR (400 MHz, $\left.\mathrm{CDCl}_{3}\right) \delta 7.95(\mathrm{~d}, J=8.2 \mathrm{~Hz}, 1 \mathrm{H}), 7.69$ (s, 2H), 7.65 (d, $J=8.2 \mathrm{~Hz}, 1 \mathrm{H}), 7.53$ (d, $J=8.2 \mathrm{~Hz}, 1 \mathrm{H})$, 7.28 (t, $J=7.8 \mathrm{~Hz}, 1 \mathrm{H}), 7.18$ (t, $J=7.8 \mathrm{~Hz}, 1 \mathrm{H}), 6.87$ (d, $J$ $=8.2 \mathrm{~Hz}, 1 \mathrm{H}), 5.37$ (s, 2H). ${ }^{13} \mathrm{C} \mathrm{NMR}\left(100 \mathrm{MHz}, \mathrm{CDCl}_{3}\right)$ $\delta$ 148.0, 138.6, 136.7, 135.4, 134.0, 133.2, 132.7, 130.0, 129.1 (q, $J=5.4 \mathrm{~Hz}), 128.5,128.2,125.8,124.5$ (q, $J=$ $34.5 \mathrm{~Hz}$ ), 122.0, 121.5 (q, $J=273.0 \mathrm{~Hz}), 111.1,54.9$. $\mathrm{C}_{17} \mathrm{H}_{10} \mathrm{BrClF}_{3} \mathrm{~N}_{3} \mathrm{O}_{2}$ (460.63): Calcd C 44.33, H 2.19; Found C 44.14, H 2.12 .

\section{1-(3-(1-(2-Bromobenzyl)-4-chloropyrazol-5-}

yl)phenyl)ethanone (8) From 1-(2-bromobenzyl)-4chloropyrazole $1 \quad(0.271 \mathrm{~g}, \quad 1 \mathrm{mmol})$ and 3bromoacetophenone $(0.299 \mathrm{~g}, 1.5 \mathrm{mmol})$ product 8 was obtained in 62\% (0.241 g) yield. ${ }^{1} \mathrm{H}$ NMR (400 MHz, $\left.\mathrm{CDCl}_{3}\right) \delta 8.00(\mathrm{dm}, J=3.8 \mathrm{~Hz}, 1 \mathrm{H}), 7.81(\mathrm{~s}, 1 \mathrm{H}), 7.67$ (s, 1H), 7.59-7.48 (m, 3H), 7.28 (t, $J=7.8 \mathrm{~Hz}, 1 \mathrm{H}), 7.15$ (t, $J$ $=7.8 \mathrm{~Hz}, 1 \mathrm{H}), 6.82(\mathrm{~d}, J=8.2 \mathrm{~Hz}, 1 \mathrm{H}), 5.34(\mathrm{~s}, 2 \mathrm{H}), 2.47$ (s, 3H). ${ }^{13} \mathrm{C}$ NMR (100 MHz, $\left.\mathrm{CDCl}_{3}\right) \delta 197.2,139.5$, $138.4,137.7,136.3,134.0,132.9,129.6,129.5,129.4$, 129.1, 128.4, 128.2, 128.1, 121.9, 109.9, 54.5, 26.6. $\mathrm{C}_{18} \mathrm{H}_{14} \mathrm{BrClN}_{2} \mathrm{O}$ (389.67): Calcd C 55.48, H 3.62; Found C 55.36, H 3.47.

5-(3,5-Bis(trifluoromethyl)phenyl)-1-(2-bromobenzyl)4-chloropyrazole (9) From 1-(2-bromobenzyl)-4chloropyrazole 1 (0.271 g, $1 \mathrm{mmol})$ and 3,5bis(trifluoromethyl)bromobenzene $(0.440 \mathrm{~g}, 1.5 \mathrm{mmol})$ product 9 was obtained in $72 \%$ (0.347 g) yield. ${ }^{1} \mathrm{H}$ NMR $\left(400 \mathrm{MHz}, \mathrm{CDCl}_{3}\right) \delta 7.92(\mathrm{~s}, 1 \mathrm{H}), 7.69(\mathrm{~s}, 1 \mathrm{H}), 7.68$ (s, 2H), 7.52 (d, $J=8.2 \mathrm{~Hz}, 1 \mathrm{H}), 7.27(\mathrm{t}, J=7.8 \mathrm{~Hz}, 1 \mathrm{H}), 7.18$ (t, $J=7.8 \mathrm{~Hz}, 1 \mathrm{H}), 6.90$ (d, $J=8.2 \mathrm{~Hz}, 1 \mathrm{H}), 5.37$ (s, 2H). ${ }^{13} \mathrm{C}$ NMR $\left(100 \mathrm{MHz}, \mathrm{CDCl}_{3}\right) \delta 138.3,137.1,135.4,133.0$, $132.3(\mathrm{q}, J=33.9 \mathrm{~Hz}), 129.8,129.7,129.6(\mathrm{~m}), 128.5$, 128.0, 122.6 (q, $J=273.0 \mathrm{~Hz}), 123.1(\mathrm{~m}), 121.9,110.7$, 54.7. $\mathrm{C}_{18} \mathrm{H}_{10} \mathrm{BrClF}_{6} \mathrm{~N}_{2}$ (483.63): Calcd C 44.70, H 2.08; Found C 44.89, H 2.32.

2-(1-(2-Bromobenzyl)-4-chloropyrazol-5-yl)benzonitrile (10) From 1-(2-bromobenzyl)-4-chloropyrazole 1 (0.271 g, $1 \mathrm{mmol})$ and 2-bromobenzonitrile (0.273 g, $1.5 \mathrm{mmol})$ product 10 was obtained in $90 \%$ (0.335 g) yield. ${ }^{1} \mathrm{H}$ NMR $\left(400 \mathrm{MHz}, \mathrm{CDCl}_{3}\right) \delta 7.78(\mathrm{~d}, J=8.2 \mathrm{~Hz}, 1 \mathrm{H}), 7.69(\mathrm{~s}, 1 \mathrm{H})$, 7.66 (t, $J=7.8 \mathrm{~Hz}, 1 \mathrm{H}), 7.57$ (t, $J=7.8 \mathrm{~Hz}, 1 \mathrm{H}), 7.45$ (d, $J$ $=8.2 \mathrm{~Hz}, 1 \mathrm{H}), 7.35(\mathrm{~d}, J=8.2 \mathrm{~Hz}, 1 \mathrm{H}), 7.26(\mathrm{t}, J=7.8 \mathrm{~Hz}$, 1H), 7.12 (t, $J=7.8 \mathrm{~Hz}, 1 \mathrm{H}$ ), 6.87 (d, $J=8.2 \mathrm{~Hz}, 1 \mathrm{H}$ ), 5.40 $(\mathrm{d}, J=16.1 \mathrm{~Hz}, 1 \mathrm{H}), 5.31$ (d, $J=16.1 \mathrm{~Hz}, 1 \mathrm{H}) .{ }^{13} \mathrm{C}$ NMR 
$\left(100 \mathrm{MHz}, \mathrm{CDCl}_{3}\right) \delta 138.4,137.0,135.6,133.8,133.3$, $132.9,131.7,131.5,130.4,129.8,129.3,128.3,122.4$, 117.1, 114.4, 112.0, 54.9. $\mathrm{C}_{17} \mathrm{H}_{11} \mathrm{BrClN}_{3}$ (372.65): Calcd C 54.79, H 2.98; Found C 54.99, H 3.14.

\section{2-(1-(2-Bromobenzyl)-4-chloropyrazol-5-}

yl)benzaldehyde (11) From 1-(2-bromobenzyl)-4chloropyrazole $1 \quad(0.271 \mathrm{~g}, \quad 1 \mathrm{mmol})$ and 2bromobenzaldehyde (0.278 g, $1.5 \mathrm{mmol})$ product 11 was obtained in $43 \%$ (0.161 g) yield. ${ }^{1} \mathrm{H}$ NMR (400 MHz, $\left.\mathrm{CDCl}_{3}\right) \delta 9.71(\mathrm{~s}, 1 \mathrm{H}), 8.03(\mathrm{~d}, J=8.2 \mathrm{~Hz}, 1 \mathrm{H}), 7.70-7.58$ (m, 3H), 7.42 (d, $J=8.2 \mathrm{~Hz}, 1 \mathrm{H}), 7.30-7.27$ (m, 1H), 7.26 (t, $J=7.8 \mathrm{~Hz}, 1 \mathrm{H}), 7.12$ (t, $J=7.8 \mathrm{~Hz}, 1 \mathrm{H}), 6.87$ (d, $J=8.2$ Hz, 1H), 5.36 (d, $J=15.9 \mathrm{~Hz}, 1 \mathrm{H}), 5.23$ (d, $J=15.9 \mathrm{~Hz}$, 1H). ${ }^{13} \mathrm{C} \mathrm{NMR}\left(100 \mathrm{MHz}, \mathrm{CDCl}_{3}\right) \delta 190.0,137.6,136.4$, 135.2 , 134.5, 134.0, 132.6, 131.1, 130.3, 129.9, 129.4, 128.9, 128.6, 127.6, 122.2, 111.7, 54.3. $\mathrm{C}_{17} \mathrm{H}_{12} \mathrm{BrClN}_{2} \mathrm{O}$ (375.65): Calcd C 54.35, H 3.22; Found C 54.24, H 3.00.

\section{3-(1-(2-Bromobenzyl)-4-chloropyrazol-5-yl)pyridine}

(12) From 1-(2-bromobenzyl)-4-chloropyrazole 1 (0.271 g, $1 \mathrm{mmol})$ and 3-bromopyridine ( $0.237 \mathrm{~g}, 1.5 \mathrm{mmol})$ product 12 was obtained in $76 \%\left(0.264\right.$ g) yield. ${ }^{1} \mathrm{H}$ NMR (400 $\left.\mathrm{MHz}, \mathrm{CDCl}_{3}\right) \delta 8.68$ (bs, $\left.1 \mathrm{H}\right), 8.57$ (s, $\left.1 \mathrm{H}\right), 7.68$ (s, 1H), 7.62 (d, $J=7.7 \mathrm{~Hz}, 1 \mathrm{H}), 7.52$ (d, $J=7.9 \mathrm{~Hz}, 1 \mathrm{H}), 7.37$ (dd, $J=7.7,4.8 \mathrm{~Hz}, 1 \mathrm{H}), 7.25(\mathrm{t}, J=7.8 \mathrm{~Hz}, 1 \mathrm{H}), 7.16(\mathrm{t}, J=$ $7.8 \mathrm{~Hz}, 1 \mathrm{H}), 6.79$ (d, $J=8.2 \mathrm{~Hz}, 1 \mathrm{H}), 5.35(\mathrm{~s}, 2 \mathrm{H}) .{ }^{13} \mathrm{C}$ NMR $\left(100 \mathrm{MHz}, \mathrm{CDCl}_{3}\right) \delta 150.8,150.4,138.8,137.6$, $137.3,136.2$, 133.3, 129.9, 128.7, 128.4, 124.4, 124.0, 122.3, 110.8, 54.9. $\mathrm{C}_{15} \mathrm{H}_{11} \mathrm{BrClN}_{3}$ (348.62): Calcd C 51.68, H 3.18; Found C 51.42, H 3.30.

\section{3-(1-(2-Bromobenzyl)-4-chloropyrazol-5-yl)quinoline}

(13) From 1-(2-bromobenzyl)-4-chloropyrazole 1 (0.271 g, $1 \mathrm{mmol}$ ) and 3-bromoquinoline (0.312 g, $1.5 \mathrm{mmol})$ product 13 was obtained in $86 \%(0.342 \mathrm{~g})$ yield. ${ }^{1} \mathrm{H}$ NMR $\left(400 \mathrm{MHz}, \mathrm{CDCl}_{3}\right) \delta 8.83(\mathrm{~s}, 1 \mathrm{H}), 8.13(\mathrm{~d}, J=8.2 \mathrm{~Hz}, 1 \mathrm{H})$, 8.07 (s, 1H), 7.82-7.77 (m, 2H), 7.71 (s, 1H), 7.59 (t, $J=$ $7.8 \mathrm{~Hz}, 1 \mathrm{H}), 7.48$ (d, $J=8.2 \mathrm{~Hz}, 1 \mathrm{H}), 7.26$ (t, $J=7.8 \mathrm{~Hz}$, $1 \mathrm{H}), 7.13$ (t, $J=7.8 \mathrm{~Hz}, 1 \mathrm{H}), 6.87$ (d, $J=8.2 \mathrm{~Hz}, 1 \mathrm{H}), 5.40$ (s, 2H). ${ }^{13} \mathrm{C}$ NMR $\left(100 \mathrm{MHz}, \mathrm{CDCl}_{3}\right) \delta 150.0,148.0$, $138.5,137.4,137.1,136.0,132.9,130.9,129.6,126.5$, 128.5, 128.3, 128.0, 127.6, 127.3, 122.0, 121.0, 110.7, 54.6. $\mathrm{C}_{19} \mathrm{H}_{13} \mathrm{BrClN}_{3}$ (398.68): Calcd C 57.24, H 3.29; Found C 57.04, H 3.07.

\section{5-(1-(2-Bromobenzyl)-4-chloropyrazol-5-yl)pyrimidine}

(14) From 1-(2-bromobenzyl)-4-chloropyrazole 1 (0.271 g, $1 \mathrm{mmol})$ and 5-bromopyrimidine (0.239 g, $1.5 \mathrm{mmol})$ product 14 was obtained in $88 \%$ (0.307 g) yield. ${ }^{1} \mathrm{H}$ NMR $\left(400 \mathrm{MHz}, \mathrm{CDCl}_{3}\right) \delta 9.26(\mathrm{~s}, 1 \mathrm{H}), 8.67$ (s, 2H), $7.70(\mathrm{~s}$, 1H), 7.52 (d, $J=8.2 \mathrm{~Hz}, 1 \mathrm{H}), 7.26$ (t, $J=7.8 \mathrm{~Hz}, 1 \mathrm{H}), 7.13$ (t, $J=7.8 \mathrm{~Hz}, 1 \mathrm{H}), 6.82$ (d, $J=8.2 \mathrm{~Hz}, 1 \mathrm{H}), 5.36(\mathrm{~s}, 2 \mathrm{H})$. ${ }^{13} \mathrm{C}$ NMR $\left(100 \mathrm{MHz}, \mathrm{CDCl}_{3}\right) \delta 158.7,156.7,138.4,135.1$, 133.7, 132.9, 129.6, 128.2, 127.9, 122.6, 121.8, 111.1, 54.5. $\mathrm{C}_{14} \mathrm{H}_{10} \mathrm{BrClN}_{4}$ (349.61): Calcd C 48.10, H 2.88; Found C 48.32, H 2.71.
4-Bromo-1-(2-bromobenzyl)-5-(4-nitrophenyl)-pyrazole (15) From 1-(2-bromobenzyl)-4-bromopyrazole 2 (0.315 g, $1 \mathrm{mmol})$ and 4-bromonitrobenzene (0.303 g, $1.5 \mathrm{mmol})$ product 15 was obtained in 60\% (0.262 g) yield. ${ }^{1} \mathrm{H}$ NMR $\left(400 \mathrm{MHz}, \mathrm{CDCl}_{3}\right) \delta 8.28(\mathrm{~d}, J=8.4 \mathrm{~Hz}, 2 \mathrm{H}), 7.71(\mathrm{~s}, 1 \mathrm{H})$, 7.52 (d, $J=8.2 \mathrm{~Hz}, 1 \mathrm{H}), 7.48$ (d, $J=8.4 \mathrm{~Hz}, 2 \mathrm{H}), 7.28$ (t, $J$ $=7.8 \mathrm{~Hz}, 1 \mathrm{H}), 7.17(\mathrm{t}, J=7.8 \mathrm{~Hz}, 1 \mathrm{H}), 6.81(\mathrm{~d}, J=8.2 \mathrm{~Hz}$, $1 \mathrm{H}), 5.37$ (s, 2H). ${ }^{13} \mathrm{C} \mathrm{NMR}\left(100 \mathrm{MHz}, \mathrm{CDCl}_{3}\right) \delta 148.0$, 140.5, 139.6, 135.5, 134.3, 132.7, 130.5, 129.4, 128.1, 127.8, 123.8, 121.7, 94.9, 54.4. $\mathrm{C}_{16} \mathrm{H}_{11} \mathrm{Br}_{2} \mathrm{~N}_{3} \mathrm{O}_{2}$ (437.08): Calcd C 43.97, H 2.54; Found C 43.80, H 2.41.

\section{4-(4-Bromo-1-(2-bromobenzyl)-pyrazol-5-}

yl)benzonitrile (16) From 1-(2-bromobenzyl)-4bromopyrazole 2 (0.315 g, $1 \mathrm{mmol})$ and 4bromobenzonitrile $(0.273 \mathrm{~g}, 1.5 \mathrm{mmol})$ product 16 was obtained in 65\% (0.271 g) yield. ${ }^{1} \mathrm{H}$ NMR (400 MHz, $\left.\mathrm{CDCl}_{3}\right) \delta 7.71(\mathrm{~d}, J=8.4 \mathrm{~Hz}, 2 \mathrm{H}), 7.69(\mathrm{~s}, 1 \mathrm{H}), 7.51$ (d, $J$ $=8.2 \mathrm{~Hz}, 1 \mathrm{H}), 7.41(\mathrm{~d}, J=8.4 \mathrm{~Hz}, 2 \mathrm{H}), 7.28(\mathrm{t}, J=7.8 \mathrm{~Hz}$, $1 \mathrm{H}), 7.17$ (t, $J=7.8 \mathrm{~Hz}, 1 \mathrm{H}), 6.80$ (d, $J=8.2 \mathrm{~Hz}, 1 \mathrm{H}), 5.35$ (s, 2H). ${ }^{13} \mathrm{C}$ NMR (100 MHz, $\left.\mathrm{CDCl}_{3}\right) \delta 140.4,139.9$, 135.5, 132.7, 132.4, 132.3, 130.1, 129.3, 128.1, 127.7, 121.7, 117.9, 113.1, 94.7, 54.3. $\mathrm{C}_{17} \mathrm{H}_{11} \mathrm{Br}_{2} \mathrm{~N}_{3}$ (417.10): Calcd C 48.95, H 2.66; Found C 48.99, H 2.48.

\section{4-(4-Bromo-1-(2-bromobenzyl)-pyrazol-5-}

yl)benzaldehyde (17) From 1-(2-bromobenzyl)-4bromopyrazole 2 (0.315 g, $1 \mathrm{mmol})$ and 4bromobenzaldehyde (0.278 g, $1.5 \mathrm{mmol})$ product 17 was obtained in 63\% (0.265 g) yield. ${ }^{1} \mathrm{H}$ NMR (400 MHz, $\left.\mathrm{CDCl}_{3}\right) \delta 10.06(\mathrm{~s}, 1 \mathrm{H}), 7.94(\mathrm{~d}, J=8.4 \mathrm{~Hz}, 2 \mathrm{H}), 7.70$ (s, $1 \mathrm{H}), 7.50$ (d, $J=8.2 \mathrm{~Hz}, 1 \mathrm{H}), 7.48$ (d, $J=8.4 \mathrm{~Hz}, 2 \mathrm{H}), 7.27$ (t, $J=7.8 \mathrm{~Hz}, 1 \mathrm{H}), 7.17$ (t, $J=7.8 \mathrm{~Hz}, 1 \mathrm{H}), 6.80$ (d, $J=8.2$ $\mathrm{Hz}, 1 \mathrm{H}), 5.37$ (s, 2H). ${ }^{13} \mathrm{C} \mathrm{NMR}\left(100 \mathrm{MHz}, \mathrm{CDCl}_{3}\right) \delta$ $191.7,141.0,140.9,136.8,136.2,134.2,133.1,130.6$, 130.2, 129.7, 128.6, 128.2, 122.1, 95.0, 54.8. $\mathrm{C}_{17} \mathrm{H}_{12} \mathrm{Br}_{2} \mathrm{~N}_{2} \mathrm{O}$ (420.10): Calcd C 48.60, H 2.88; Found C 48.41, H 2.64.

\begin{abstract}
4-Bromo-1-(2-bromobenzyl)-5-(4-nitro-3(trifluoromethyl)phenyl)-pyrazole (18) From 1-(2bromobenzyl)-4-bromopyrazole 2 (0.315 g, $1 \mathrm{mmol}$ ) and 4bromo-1-nitro-2-(trifluoromethyl)benzene (0.405 g, 1.5 mmol) product 18 was obtained in $67 \%\left(0.338\right.$ g) yield. ${ }^{1} \mathrm{H}$ NMR (400 MHz, $\left.\mathrm{CDCl}_{3}\right) \delta 7.94(\mathrm{~d}, J=8.2 \mathrm{~Hz}, 1 \mathrm{H}), 7.72$ (s, 1H), 7.67 (s, 1H), 7.63 (d, $J=8.2 \mathrm{~Hz}, 1 \mathrm{H}), 7.53$ (d, $J=$ $8.2 \mathrm{~Hz}, 1 \mathrm{H}), 7.28$ (t, $J=7.8 \mathrm{~Hz}, 1 \mathrm{H}), 7.18$ (t, $J=7.8 \mathrm{~Hz}$, $1 \mathrm{H}), 6.88(\mathrm{~d}, J=8.2 \mathrm{~Hz}, 1 \mathrm{H}), 5.38(\mathrm{~s}, 2 \mathrm{H}) .{ }^{13} \mathrm{C}$ NMR $(100$ $\left.\mathrm{MHz}, \mathrm{CDCl}_{3}\right) \delta 148.4,141.1,138.7,135.7,134.6,133.5$, 133.4, 130.3, 129.7 (q, $J=5.3 \mathrm{~Hz}), 128.9,128.5,126.1$, 124.8 (q, $J=34.6 \mathrm{~Hz}), 122.4,121.9$ (q, $J=274.0 \mathrm{~Hz}$ ), 96.1, 55.3. $\mathrm{C}_{17} \mathrm{H}_{10} \mathrm{Br}_{2} \mathrm{~F}_{3} \mathrm{~N}_{3} \mathrm{O}_{2}$ (505.08): Calcd C 40.43, H 2.00; Found C 40.54, H 1.88 .
\end{abstract}

1-(3-(4-Bromo-1-(2-bromobenzyl)-pyrazol-5yl)phenyl)ethanone (19) From 1-(2-bromobenzyl)-4- 
bromopyrazole 2 (0.315 g, $1 \mathrm{mmol})$ and 3bromoacetophenone (0.299 g, $1.5 \mathrm{mmol})$ product 19 was obtained in 44\% (0.191 g) yield. ${ }^{1} \mathrm{H}$ NMR (400 MHz, $\left.\mathrm{CDCl}_{3}\right) \delta 8.01(\mathrm{~d}, J=8.2 \mathrm{~Hz}, 1 \mathrm{H}), 7.80(\mathrm{~s}, 1 \mathrm{H}), 7.69$ (s, 1H), 7.57-7.47 (m, 3H), 7.26 (t, $J=7.8 \mathrm{~Hz}, 1 \mathrm{H}), 7.15$ (t, $J$ $=7.8 \mathrm{~Hz}, 1 \mathrm{H}), 6.83(\mathrm{~d}, J=8.2 \mathrm{~Hz}, 1 \mathrm{H}), 5.35$ (s, 2H), 2.47 (s, 3H). ${ }^{13} \mathrm{C}$ NMR $\left(100 \mathrm{MHz}, \mathrm{CDCl}_{3}\right) \delta 197.5,141.4$, $140.8,137.9,136.6,134.5,133.2,130.1,129.8,129.7$, 129.5, 129.0, 128.8, 128.4, 122.2, 94.9, 54.9, 26.9. $\mathrm{C}_{18} \mathrm{H}_{14} \mathrm{Br}_{2} \mathrm{~N}_{2} \mathrm{O}$ (434.12): Calcd $\mathrm{C}$ 49.80, $\mathrm{H}$ 3.25; Found $\mathrm{C}$ 49.99, H 3.35.

\section{5-(3,5-Bis(trifluoromethyl)phenyl)-4-bromo-1-(2-}

bromobenzyl)-pyrazole (20) From 1-(2-bromobenzyl)-4bromopyrazole 2 (0.315 g, $1 \mathrm{mmol})$ and 3,5bis(trifluoromethyl)bromobenzene $(0.440 \mathrm{~g}, 1.5 \mathrm{mmol})$ product 20 was obtained in $81 \%$ (0.427 g) yield. ${ }^{1} \mathrm{H}$ NMR $\left(400 \mathrm{MHz}, \mathrm{CDCl}_{3}\right) \delta 7.92(\mathrm{~s}, 1 \mathrm{H}), 7.70(\mathrm{~s}, 1 \mathrm{H}), 7.66(\mathrm{~s}$, 2H), 7.49 (d, $J=8.2 \mathrm{~Hz}, 1 \mathrm{H}), 7.26$ (t, $J=7.8 \mathrm{~Hz}, 1 \mathrm{H}), 7.15$ (t, $J=7.8 \mathrm{~Hz}, 1 \mathrm{H}), 6.89$ (d, $J=8.2 \mathrm{~Hz}, 1 \mathrm{H}), 5.37(\mathrm{~s}, 2 \mathrm{H})$. ${ }^{13} \mathrm{C}$ NMR (100 MHz, $\left.\mathrm{CDCl}_{3}\right) \delta 140.5,138.9,135.5,133.1$, 132.5 (q, $J=33.9 \mathrm{~Hz}), 130.4,130.0(\mathrm{~m}), 129.9,128.7$, 128.1, $123.3(\mathrm{~m}), 123.0(\mathrm{q}, J=273.0 \mathrm{~Hz}), 122.1,95.5$, 54.9. $\mathrm{C}_{18} \mathrm{H}_{10} \mathrm{Br}_{2} \mathrm{~F}_{6} \mathrm{~N}_{2}$ (528.08): Calcd $\mathrm{C}$ 40.94, $\mathrm{H}$ 1.91; Found C 41.10, H 2.04.

2-(4-Bromo-1-(2-bromobenzyl)-pyrazol-5-

yl)benzonitrile (21) From 1-(2-bromobenzyl)-4bromopyrazole 2 (0.315 g, $1 \mathrm{mmol})$ and 2bromobenzonitrile $(0.273 \mathrm{~g}, 1.5 \mathrm{mmol})$ product 21 was obtained in $77 \%$ (0.321 g) yield. ${ }^{1} \mathrm{H}$ NMR (400 MHz, $\left.\mathrm{CDCl}_{3}\right) \delta 7.79(\mathrm{~d}, J=8.2 \mathrm{~Hz}, 1 \mathrm{H}), 7.69(\mathrm{~s}, 1 \mathrm{H}), 7.66(\mathrm{t}, J=$ $7.8 \mathrm{~Hz}, 1 \mathrm{H}), 7.57$ (t, $J=7.8 \mathrm{~Hz}, 1 \mathrm{H}), 7.45$ (d, $J=8.2 \mathrm{~Hz}$, $1 \mathrm{H}), 7.35$ (d, $J=8.2 \mathrm{~Hz}, 1 \mathrm{H}), 7.27$ (t, $J=7.8 \mathrm{~Hz}, 1 \mathrm{H}), 7.13$ (t, $J=7.8 \mathrm{~Hz}, 1 \mathrm{H}), 6.89$ (d, $J=8.2 \mathrm{~Hz}, 1 \mathrm{H}), 5.42$ (d, $J=$ $16.1 \mathrm{~Hz}, 1 \mathrm{H}), 5.33$ (d, $J=16.1 \mathrm{~Hz}, 1 \mathrm{H}) .{ }^{13} \mathrm{C}$ NMR $(100$ $\left.\mathrm{MHz}, \mathrm{CDCl}_{3}\right) \delta 140.4,135.4,133.6,133.1,132.8,131.9$, 131.6, 130.3, 129.7, 129.2, 128.1, 122.3, 116.9, 114.4, 96.6, 54.8. $\mathrm{C}_{17} \mathrm{H}_{11} \mathrm{Br}_{2} \mathrm{~N}_{3}$ (417.10): Calcd C 48.95, H 2.66; Found C 48.87, H 2.70.

\section{2-(4-Bromo-1-(2-bromobenzyl)-pyrazol-5-}

yl)benzaldehyde (22) From 1-(2-bromobenzyl)-4bromopyrazole 2 (0.315 g, $1 \mathrm{mmol})$ and 2bromobenzaldehyde $(0.278 \mathrm{~g}, 1.5 \mathrm{mmol})$ product 22 was obtained in 55\% (0.231 g) yield. ${ }^{1} \mathrm{H}$ NMR (400 MHz, $\left.\mathrm{CDCl}_{3}\right) \delta 9.68(\mathrm{~s}, 1 \mathrm{H}), 8.02(\mathrm{~d}, J=8.2 \mathrm{~Hz}, 1 \mathrm{H}), 7.70$ (s, 1H), 7.69-7.58 (m, 2H), 7.42 (d, $J=8.2 \mathrm{~Hz}, 1 \mathrm{H}), 7.30-7.27$ (m, 1H), 7.26 (t, $J=7.8 \mathrm{~Hz}, 1 \mathrm{H}), 7.12$ (t, $J=7.8 \mathrm{~Hz}, 1 \mathrm{H})$, 6.87 (d, $J=8.2 \mathrm{~Hz}, 1 \mathrm{H}), 5.37$ (d, $J=15.9 \mathrm{~Hz}, 1 \mathrm{H}), 5.24$ (d, $J=15.9 \mathrm{~Hz}, 1 \mathrm{H}) .{ }^{13} \mathrm{C} \mathrm{NMR}\left(100 \mathrm{MHz}, \mathrm{CDCl}_{3}\right) \delta 190.0$, 139.8, 138.3, 135.3, 134.6, 134.1, 132.8, 131.3, 130.7, 130.4, 129.6, 129.1, 128.7, 127.8, 122.3, 96.9, 54.5. $\mathrm{C}_{17} \mathrm{H}_{12} \mathrm{Br}_{2} \mathrm{~N}_{2} \mathrm{O}$ (420.10): Calcd C 48.60, H 2.88; Found $\mathrm{C}$ 48.78, H 2.98.
3-(4-Bromo-1-(2-bromobenzyl)-pyrazol-5-yl)pyridine (23) From 1-(2-bromobenzyl)-4-bromopyrazole 2 (0.315 g, $1 \mathrm{mmol}$ ) and 3-bromopyridine $(0.237 \mathrm{~g}, 1.5 \mathrm{mmol})$ product 23 was obtained in $42 \%$ (0.165 g) yield. ${ }^{1} \mathrm{H}$ NMR (400 $\left.\mathrm{MHz}, \mathrm{CDCl}_{3}\right) \delta 8.68$ (bs, $\left.1 \mathrm{H}\right), 8.56$ (bs, $1 \mathrm{H}$ ), 7.70 (s, 1H), 7.61 (d, $J=7.7 \mathrm{~Hz}, 1 \mathrm{H}), 7.50$ (d, $J=7.9 \mathrm{~Hz}, 1 \mathrm{H}$ ), 7.37 (dd, $J=7.7,4.8 \mathrm{~Hz}, 1 \mathrm{H}), 7.25$ (t, $J=7.8 \mathrm{~Hz}, 1 \mathrm{H}), 7.14$ (t, $J=$ $7.8 \mathrm{~Hz}, 1 \mathrm{H}), 6.78(\mathrm{~d}, J=8.2 \mathrm{~Hz}, 1 \mathrm{H}), 5.35(\mathrm{~s}, 2 \mathrm{H}) .{ }^{13} \mathrm{C}$ NMR $\left(100 \mathrm{MHz}, \mathrm{CDCl}_{3}\right) \delta 150.8,150.5,141.0,139.3$, 137.6, 136.3, 133.3, 129.9, 128.8, 128.4, 125.0, 124.0, 122.4, 95.6, 54.9. $\mathrm{C}_{15} \mathrm{H}_{11} \mathrm{Br}_{2} \mathrm{~N}_{3}$ (393.07): Calcd C 45.83, $\mathrm{H}$ 2.82; Found C 45.71, H 2.59.

\section{3-(4-Bromo-1-(2-bromobenzyl)-pyrazol-5-yl)quinoline}

(24) From 1-(2-bromobenzyl)-4-bromopyrazole 2 (0.315 g, $1 \mathrm{mmol}$ ) and 3-bromoquinoline (0.312 g, $1.5 \mathrm{mmol})$ product 24 was obtained in 56\% (0.248 g) yield. ${ }^{1} \mathrm{H}$ NMR $\left(400 \mathrm{MHz}, \mathrm{CDCl}_{3}\right) \delta 8.83(\mathrm{~s}, 1 \mathrm{H}), 8.14(\mathrm{~d}, J=8.2 \mathrm{~Hz}, 1 \mathrm{H})$, 8.07 (s, 1H), 7.82-7.77 (m, 2H), $7.71(\mathrm{~s}, 1 \mathrm{H}), 7.59(\mathrm{t}, J=$ $7.8 \mathrm{~Hz}, 1 \mathrm{H}), 7.48(\mathrm{~d}, J=8.2 \mathrm{~Hz}, 1 \mathrm{H}), 7.26(\mathrm{t}, J=7.8 \mathrm{~Hz}$, $1 \mathrm{H}), 7.13$ (t, $J=7.8 \mathrm{~Hz}, 1 \mathrm{H}), 6.87$ (d, $J=8.2 \mathrm{~Hz}, 1 \mathrm{H}), 5.41$ (s, 2H). ${ }^{13} \mathrm{C}$ NMR (100 MHz, $\left.\mathrm{CDCl}_{3}\right) \delta 150.4,148.3$, $140.9,139.4,137.6,136.3,133.2,131.2,129.9,129.8$, 128.8, 128.6, 128.4, 127.9, 127.6, 122.4, 121.9, 95.8, 55.0. $\mathrm{C}_{19} \mathrm{H}_{13} \mathrm{Br}_{2} \mathrm{~N}_{3}$ (443.13): Calcd C 51.50, H 2.96; Found C 51.31, H 2.74 .

5-(4-Bromo-1-(2-bromobenzyl)-pyrazol-5-yl)pyrimidine (25) From 1-(2-bromobenzyl)-4-bromopyrazole 2 (0.315 g, $1 \mathrm{mmol})$ and 5-bromopyrimidine (0.239 g, $1.5 \mathrm{mmol})$ product 25 was obtained in $74 \%(0.291 \mathrm{~g})$ yield. ${ }^{1} \mathrm{H}$ NMR $\left(400 \mathrm{MHz}, \mathrm{CDCl}_{3}\right) \delta 9.26(\mathrm{~s}, 1 \mathrm{H}), 8.66(\mathrm{~s}, 2 \mathrm{H}), 7.72(\mathrm{~s}$, 1H), 7.52 (d, $J=8.2 \mathrm{~Hz}, 1 \mathrm{H}), 7.26$ (t, $J=7.8 \mathrm{~Hz}, 1 \mathrm{H}), 7.13$ (t, $J=7.8 \mathrm{~Hz}, 1 \mathrm{H}), 6.82$ (d, $J=8.2 \mathrm{~Hz}, 1 \mathrm{H}), 5.37$ (s, 2H). ${ }^{13} \mathrm{C}$ NMR $\left(100 \mathrm{MHz}, \mathrm{CDCl}_{3}\right) \delta 158.8,156.9,140.5,135.4$, 135.1, 132.8, 129.6, 128.3, 127.9, 123.1, 121.8, 95.8, 54.5. $\mathrm{C}_{14} \mathrm{H}_{10} \mathrm{Br}_{2} \mathrm{~N}_{4}$ (394.06): Calcd C 42.67, H 2.56; Found $\mathrm{C}$ 42.78, H 2.40 .

General procedure for the preparation of 26-29: The reaction of the 1-(2-bromobenzyl)-4-chloro-5-arylpyrazole derivative $(1 \mathrm{mmol})$, heteroarene $(1.5 \mathrm{mmol})$, and KOAc (0.196 g, $2 \mathrm{mmol})$ at $150^{\circ} \mathrm{C}$ during $20 \mathrm{~h}$ in DMA (4 mL) in the presence of $\mathrm{PdCl}\left(\mathrm{C}_{3} \mathrm{H}_{5}\right)(\mathrm{dppb})(12.2 \mathrm{mg}, 0.02 \mathrm{mmol})$ under argon affords the coupling product after evaporation of the solvent and purification on silica gel.

\section{5-(2-((4-Chloro-5-(4-nitrophenyl)-pyrazol-1-}

yl)methyl)phenyl)-2-ethyl-4-methylthiazole (26) From 1(2-bromobenzyl)-4-chloro-5-(4-nitrophenyl)-pyrazole 4 (0.392 g, $1 \mathrm{mmol}$ ) and 2-ethyl-4-methylthiazole (0.191 g, $1.5 \mathrm{mmol}$ ) product 26 was obtained in 89\% (0.390 g) yield. ${ }^{1} \mathrm{H}$ NMR (400 MHz, $\mathrm{CDCl}_{3}$ ) $\delta 8.22(\mathrm{~d}, J=8.2 \mathrm{~Hz}, 2 \mathrm{H})$, 7.62 (s, 1H), 7.40-7.20 (m, 5H), 6.97 (d, $J=8.2 \mathrm{~Hz}, 1 \mathrm{H})$, 5.17 (s, 2H), 2.94 (q, $J=7.6 \mathrm{~Hz}, 2 \mathrm{H}), 2.03$ (s, 3H), 1.35 (t, $J=7.6 \mathrm{~Hz}, 3 \mathrm{H}) .{ }^{13} \mathrm{C} \mathrm{NMR}\left(100 \mathrm{MHz}, \mathrm{CDCl}_{3}\right) \delta 172.0$, 149.2, 148.2, 138.5, 138.0, 136.2, 134.1, 132.1, 130.6, 
130.2, 129.5, 128.3, 127.7, 127.1, 124.1, 110.6, 52.9, 27.1, 15.6, 14.3. $\quad \mathrm{C}_{22} \mathrm{H}_{19} \mathrm{ClN}_{4} \mathrm{O}_{2} \mathrm{~S}$ (438.93): Calcd C 60.20, $\mathrm{H}$ 4.36; Found C 60.34, H 4.18.

4-Chloro-1-(2-(5-methylthiophen-2-yl)benzyl)-5-(4nitrophenyl)-pyrazole (27) From 1-(2-bromobenzyl)-4chloro-5-(4-nitrophenyl)-pyrazole 4 (0.392 g, $1 \mathrm{mmol})$ and 2-methylthiophene $(0.147 \mathrm{~g}, 1.5 \mathrm{mmol})$ product 27 was obtained in $86 \%$ (0.352 g) yield. ${ }^{1} \mathrm{H}$ NMR (400 MHz, $\left.\mathrm{CDCl}_{3}\right) \delta 8.17(\mathrm{~d}, J=8.2 \mathrm{~Hz}, 2 \mathrm{H}), 7.64(\mathrm{~s}, 1 \mathrm{H}), 7.34-7.24$ (m, 5H), 7.00 (t, $J=7.8 \mathrm{~Hz}, 1 \mathrm{H}), 6.66-6.62$ (m, 1H), 6.56 (d, $J=3.3 \mathrm{~Hz}, 1 \mathrm{H}), 5.42$ (s, 2H), 2.47 (s, 3H). ${ }^{13} \mathrm{C}$ NMR $\left(100 \mathrm{MHz}, \mathrm{CDCl}_{3}\right) \delta 147.8,140.7,138.0,137.9,137.5$, $134.2,133.8,133.1,130.8,130.1,128.1,127.8,127.3$, 126.8, 125.3, 123.6, 110.1, 52.3, 15.1. $\mathrm{C}_{21} \mathrm{H}_{16} \mathrm{ClN}_{3} \mathrm{O}_{2} \mathrm{~S}$ (409.89): Calcd C 61.53, H 3.93; Found C 61.64, H 3.75.

\section{2-(4-Chloro-1-(2-(2-ethyl-4-methylthiazol-5-yl)benzyl)- pyrazol-5-yl)benzonitrile (28) From 2-(1-(2- bromobenzyl)-4-chloropyrazol-5-yl)benzonitrile 10 (0.373 g, $1 \mathrm{mmol})$ and 2-ethyl-4-methylthiazole (0.191 g, 1.5 mmol) product 28 was obtained in $90 \%\left(0.376\right.$ g) yield. ${ }^{1} \mathrm{H}$ NMR (400 MHz, $\left.\mathrm{CDCl}_{3}\right) \delta 7.72(\mathrm{~d}, J=8.2 \mathrm{~Hz}, 1 \mathrm{H}), 7.62$ (s, 1H), 7.58 (t, $J=7.8 \mathrm{~Hz}, 1 \mathrm{H}), 7.52$ (t, $J=7.8 \mathrm{~Hz}, 1 \mathrm{H})$, 7.33 (t, $J=7.8 \mathrm{~Hz}, 1 \mathrm{H}), 7.27$ (t, $J=7.8 \mathrm{~Hz}, 1 \mathrm{H}), 7.14$ (d, $J$ $=8.2 \mathrm{~Hz}, 1 \mathrm{H}), 7.11(\mathrm{~d}, J=8.2 \mathrm{~Hz}, 1 \mathrm{H}), 6.92(\mathrm{~d}, J=8.4 \mathrm{~Hz}$, $1 \mathrm{H}), 5.19$ (d, $J=15.9 \mathrm{~Hz}, 1 \mathrm{H}), 5.06$ (d, $J=15.9 \mathrm{~Hz}, 1 \mathrm{H})$, 2.96 (q, $J=7.6 \mathrm{~Hz}, 2 \mathrm{H}), 1.95$ (s, 3H), 1.37 (t, $J=7.6 \mathrm{~Hz}$, $3 \mathrm{H}) .{ }^{13} \mathrm{C} \mathrm{NMR}\left(100 \mathrm{MHz}, \mathrm{CDCl}_{3}\right) \delta 171.4,148.6,137.7$, 136.2, 135.6, 133.2, 132.6, 131.4, 131.1, 130.9, 129.7, 129.6, 129.1, 127.7, 127.5, 126.8, 116.5, 113.8, 111.4, 52.4, 26.7, 15.0, 13.9. $\mathrm{C}_{23} \mathrm{H}_{19} \mathrm{ClN}_{4} \mathrm{~S}$ (418.94): Calcd C 65.94, H 4.57; Found C 65.87, H 4.41.}

\section{3-(1-(2-(5-Butylfuran-2-yl)benzyl)-4-chloropyrazol-5-}

yl)pyridine (29) From 3-(1-(2-bromobenzyl)-4chloropyrazol-5-yl)pyridine 12 (0.348 g, $1 \mathrm{mmol})$ and 2butylfuran $(0.186 \mathrm{~g}, 1.5 \mathrm{mmol})$ product 29 was obtained in $83 \%\left(0.324\right.$ g) yield. ${ }^{1} \mathrm{H}$ NMR (400 $\left.\mathrm{MHz}, \mathrm{CDCl}_{3}\right) \delta 8.60$ (bs, $1 \mathrm{H}), 8.56$ (bs, $1 \mathrm{H}), 7.67$ (s, 1H), 7.54 (d, $J=7.7 \mathrm{~Hz}$, $1 \mathrm{H}), 7.51$ (d, $J=7.9 \mathrm{~Hz}, 1 \mathrm{H}), 7.35-7.24$ (m, 2H), 7.20 (t, $J$ $=7.8 \mathrm{~Hz}, 1 \mathrm{H}), 6.72(\mathrm{~d}, J=8.2 \mathrm{~Hz}, 1 \mathrm{H}), 6.30(\mathrm{~d}, J=3.2 \mathrm{~Hz}$, $1 \mathrm{H}), 6.02$ (d, $J=3.2 \mathrm{~Hz}, 1 \mathrm{H}), 5.52$ (s, 2H), 2.61 (t, $J=7.6$ $\mathrm{Hz}, 2 \mathrm{H}$ ), 1.60 (quint., $J=7.6 \mathrm{~Hz}, 2 \mathrm{H}$ ), 1.34 (sext., $J=7.6$ $\mathrm{Hz}, 2 \mathrm{H}), 0.91$ (t, $J=7.6 \mathrm{~Hz}, 3 \mathrm{H}) .{ }^{13} \mathrm{C} \mathrm{NMR}(100 \mathrm{MHz}$, $\left.\mathrm{CDCl}_{3}\right) \delta 156.7,150.4,149.9,149.7,138.1,137.9,136.8$, $136.5,132.9,128.8,127.7,127.6,127.4,126.5,110.0$, 109.4, 106.5, 52.7, 29.9, 27.6, 22.0, 13.6. $\mathrm{C}_{23} \mathrm{H}_{22} \mathrm{ClN}_{3} \mathrm{O}$ (391.89): Calcd C 70.49, H 5.66; Found C 70.71, H 5.47.

\section{2-(1-(Biphenyl-2-ylmethyl)-4-chloropyrazol-5-}

yl)benzonitrile (30) The reaction of 2-(1-(2bromobenzyl)-4-chloropyrazol-5-yl)benzonitrile 10 (0.372 g, $1 \mathrm{mmol})$, phenylboronic acid (0.183 g, $1.5 \mathrm{mmol})$ and $\mathrm{K}_{2} \mathrm{CO}_{3}(0.414 \mathrm{~g}, 3 \mathrm{mmol})$ at $110^{\circ} \mathrm{C}$ during $15 \mathrm{~h}$ in DMA (4 $\mathrm{mL})$ in the presence of $\mathrm{Pd}(\mathrm{OAc})_{2}(4.5 \mathrm{mg}, 0.02 \mathrm{mmol})$ under argon affords, after evaporation of the solvent and purification on silica gel, product 30 in $88 \%$ (0.325 g) yield. ${ }^{1} \mathrm{H}$ NMR (400 MHz, $\left.\mathrm{CDCl}_{3}\right) \delta$ 7.68-7.63 (m, 1H), 7.60 (s, 1H), 7.53-7.47 (m, 2H), 7.35-7.22 (m, 5H), 7.137.09 (m, 1H), 7.04-6.90 (m, 4H), 5.25 (d, $J=16.1 \mathrm{~Hz}, 1 \mathrm{H})$, $5.16(\mathrm{~d}, J=16.1 \mathrm{~Hz}, 1 \mathrm{H}) .{ }^{13} \mathrm{C} \mathrm{NMR}\left(100 \mathrm{MHz}, \mathrm{CDCl}_{3}\right) \delta$ 140.9 , 139.9, 137.7, 136.3, 133.5, 132.8, 131.4, 131.3, 130.0, 129.8, 129.0, 128.4, 128.2, 127.9, 127.8, 127.5, 116.8, 114.1, 111.6, 52.7. $\mathrm{C}_{23} \mathrm{H}_{16} \mathrm{ClN}_{3}$ (369.84): Calcd C 74.69, H 4.36; Found C 74.80, H 4.30.

\section{4-(1-(Biphenyl-2-ylmethyl)-4-bromopyrazol-5-}

yl)benzonitrile (31) The reaction of 4-(4-bromo-1-(2bromobenzyl)-pyrazol-5-yl)benzonitrile 16 (0.417 g, 1 mmol), phenylboronic acid (0.183 g, $1.5 \mathrm{mmol})$ and $\mathrm{K}_{2} \mathrm{CO}_{3}$ (0.414 g, $3 \mathrm{mmol}$ ) at $110^{\circ} \mathrm{C}$ during $15 \mathrm{~h}$ in DMA (4 mL) in the presence of $\mathrm{Pd}(\mathrm{OAc})_{2}$ (4.5 $\mathrm{mg}, 0.02 \mathrm{mmol}$ ) under argon affords, after evaporation of the solvent and purification on silica gel, product 31 in $77 \%$ (0.319 g) yield. ${ }^{1} \mathrm{H}$ NMR $\left(400 \mathrm{MHz}, \mathrm{CDCl}_{3}\right) \delta 7.63(\mathrm{~s}, 1 \mathrm{H}), 7.58(\mathrm{~d}, J=7.6 \mathrm{~Hz}, 2 \mathrm{H})$, 7.38-7.27 (m, 5H), 7.20-7.13 (m, 3H), 7.06-6.96 (m, 3H), 5.23 (s, 2H). ${ }^{13} \mathrm{C}$ NMR (100 MHz, $\left.\mathrm{CDCl}_{3}\right) \delta 140.9,140.2$, $139.9,139.7,133.7,132.9,132.4,130.4,130.2,129.0$, 128.5, 128.1, 128.0, 127.6, 127.5, 118.3, 113.0, 94.8, 52.7. $\mathrm{C}_{23} \mathrm{H}_{16} \mathrm{BrN}_{3}$ (414.30): Calcd C 66.68, H 3.89; Found $\mathrm{C}$ 66.49, H 4.04 .

\section{3-(1-(Biphenyl-2-ylmethyl)-4-bromopyrazol-5-}

yl)quinoline (32) The reaction of 3-(4-bromo-1-(2bromobenzyl)-pyrazol-5-yl)quinoline 24 (0.443 g, $1 \mathrm{mmol})$, phenylboronic acid $(0.183 \mathrm{~g}, 1.5 \mathrm{mmol})$ and $\mathrm{K}_{2} \mathrm{CO}_{3}(0.414$ $\mathrm{g}, 3 \mathrm{mmol})$ at $110^{\circ} \mathrm{C}$ during $15 \mathrm{~h}$ in DMA (4 mL) in the presence of $\mathrm{Pd}(\mathrm{OAc})_{2}(4.5 \mathrm{mg}, 0.02 \mathrm{mmol})$ under argon affords, after evaporation of the solvent and purification on silica gel, product 32 in $84 \%$ (0.370 g) yield. ${ }^{1} \mathrm{H}$ NMR $\left(400 \mathrm{MHz}, \mathrm{CDCl}_{3}\right) \delta 8.58(\mathrm{bs}, 1 \mathrm{H}), 8.14(\mathrm{~d}, J=8.2 \mathrm{~Hz}$, 1H), 7.81 (t, $J=7.8 \mathrm{~Hz}, 1 \mathrm{H}), 7.76$ (s, 1H), 7.68 (t, $J=7.8$ $\mathrm{Hz}, 1 \mathrm{H}), 7.67$ (s, $1 \mathrm{H}), 7.61(\mathrm{t}, J=7.8 \mathrm{~Hz}, 1 \mathrm{H}), 7.35-7.27$ (m, 2H), 7.20-7.05 (m, 5H), 6.84 (d, $J=8.2 \mathrm{~Hz}, 2 \mathrm{H}), 5.27$ (s, 2H). ${ }^{13} \mathrm{C}$ NMR $\left(100 \mathrm{MHz}, \mathrm{CDCl}_{3}\right) \delta 150.4,148.0$, $141.1,140.2$, 139.9, 138.8, 137.3, 134.1, 130.9, 130.3, 129.7, 128.9, 128.4, 128.3, 128.2, 128.1, 127.8, 127.6, 127.5, 127.4, 121.9, 95.6, 52.9. $\mathrm{C}_{25} \mathrm{H}_{18} \mathrm{BrN}_{3}$ (440.33): Calcd C 68.19, H 4.12; Found C 68.00, H 4.05.

3-Chloropyrazolo[5,1-a]isoindole (33) The reaction of 1(2-bromobenzyl)-4-chloropyrazole $\mathbf{1}$ (0.271 g, $1 \mathrm{mmol}$ ) and KOAc (0.196 g, $2 \mathrm{mmol})$ at $150^{\circ} \mathrm{C}$ during $20 \mathrm{~h}$ in DMA (4 $\mathrm{mL}$ ) in the presence of $\mathrm{PdCl}\left(\mathrm{C}_{3} \mathrm{H}_{5}\right)(\mathrm{dppb})$ (12.2 mg, 0.02 mmol) under argon affords, after evaporation of the solvent and purification on silica gel, product 33 in $88 \%$ (0.167 g) yield. ${ }^{1} \mathrm{H}$ NMR (400 MHz, $\left.\mathrm{CDCl}_{3}\right) \delta 7.78(\mathrm{~d}, J=8.2 \mathrm{~Hz}$, $1 \mathrm{H}), 7.53$ (s, 1H), 7.48-7.40 (m, 2H), 7.36 (t, $J=7.8 \mathrm{~Hz}$, 1H), 5.09 (s, 2H). ${ }^{13} \mathrm{C}$ NMR $\left(100 \mathrm{MHz}, \mathrm{CDCl}_{3}\right) \delta 142.7$, 142.1, 140.1, 130.0, 128.7, 128.0, 123.7, 120.9, 102.1, 53.2. $\mathrm{C}_{10} \mathrm{H}_{7} \mathrm{ClN}_{2}$ (190.63): Calcd C 63.01, H 3.70; Found C 62.89, H 3.71 . 
3-Bromopyrazolo[5,1-a]isoindole (34) The reaction of 1(2-bromobenzyl)-4-bromopyrazole 2 (0.315 g, $1 \mathrm{mmol}$ ) and KOAc (0.196 g, $2 \mathrm{mmol})$ at $150^{\circ} \mathrm{C}$ during $20 \mathrm{~h}$ in DMA (4 $\mathrm{mL})$ in the presence of $\mathrm{PdCl}\left(\mathrm{C}_{3} \mathrm{H}_{5}\right)(\mathrm{dppb})(12.2 \mathrm{mg}, 0.02$ mmol) under argon affords, after evaporation of the solvent and purification on silica gel, product 34 in $75 \%$ (0.176 g) yield. ${ }^{1} \mathrm{H}$ NMR $\left(400 \mathrm{MHz}, \mathrm{CDCl}_{3}\right) \delta 7.81(\mathrm{~d}, J=8.2 \mathrm{~Hz}$, 1H), 7.56 (s, 1H), 7.48-7.40 (m, 2H), 7.37 (t, $J=7.8 \mathrm{~Hz}$, $1 \mathrm{H}), 5.10(\mathrm{~s}, 2 \mathrm{H}) .{ }^{13} \mathrm{C} \mathrm{NMR}\left(100 \mathrm{MHz}, \mathrm{CDCl}_{3}\right) \delta 144.4$, 144.1, 140.3, 130.2, 128.7, 128.1, 123.7, 120.7, 84.9, 53.1. $\mathrm{C}_{10} \mathrm{H}_{7} \mathrm{BrN}_{2}$ (235.08): Calcd C 51.09, H 3.00; Found C 51.24, H 3.17.

1-(2-Bromobenzyl)-4-phenylpyrazole (36) ${ }^{6}$ The reaction of 1-(2-bromobenzyl)-4-iodopyrazole 3 (0.726 g, $2 \mathrm{mmol}$ ), phenylboronic acid $(0.244 \mathrm{~g}, 2 \mathrm{mmol})$ and $\mathrm{K}_{2} \mathrm{CO}_{3}(0.828 \mathrm{~g}$, $6 \mathrm{mmol})$ at $110^{\circ} \mathrm{C}$ during $15 \mathrm{~h}$ in DMA (4 mL) in the presence of $\mathrm{PdCl}\left(\mathrm{C}_{3} \mathrm{H}_{5}\right)(\mathrm{dppb})(12.2 \mathrm{mg}, 0.04 \mathrm{mmol})$ under argon affords, after evaporation of the solvent and purification on silica gel, product 36 in $54 \%$ (0.338 g) yield. ${ }^{1} \mathrm{H}$ NMR (400 MHz, $\left.\mathrm{CDCl}_{3}\right) \delta 7.86(\mathrm{~s}, 1 \mathrm{H}), 7.71$ (s, 1H), 7.60 (d, $J=8.3 \mathrm{~Hz}, 1 \mathrm{H}), 7.48$ (d, $J=8.3 \mathrm{~Hz}, 2 \mathrm{H}), 7.36$ (t, $J=8.0 \mathrm{~Hz}, 2 \mathrm{H}), 7.31-7.15$ (m, 3H), 7.03 (d, $J=8.3 \mathrm{~Hz}$, $1 \mathrm{H}), 5.45$ (s, $2 \mathrm{H}) .{ }^{13} \mathrm{C} \mathrm{NMR}\left(100 \mathrm{MHz}, \mathrm{CDCl}_{3}\right) \delta 137.2$, $135.8,132.7,132.2,129.5,129.4,128.7,127.8,126.6$, 126.3, 125.4, 123.4, 122.8, 55.8 .

1-(2-Bromobenzyl)-4-(thiophen-2-yl)-pyrazole (37) The reaction of 1-(2-bromobenzyl)-4-iodopyrazole 3 (0.726 g, 2 mmol), 2-thienylboronic acid (0.256 g, $2 \mathrm{mmol})$ and $\mathrm{K}_{2} \mathrm{CO}_{3}$ $(0.828 \mathrm{~g}, 6 \mathrm{mmol})$ at $110^{\circ} \mathrm{C}$ during $15 \mathrm{~h}$ in DMA $(4 \mathrm{~mL})$ in the presence of $\mathrm{PdCl}\left(\mathrm{C}_{3} \mathrm{H}_{5}\right)(\mathrm{dppb})(12.2 \mathrm{mg}, 0.04 \mathrm{mmol})$ under argon affords, after evaporation of the solvent and purification on silica gel, product 37 in $62 \%$ (0.395 g) yield. ${ }^{1} \mathrm{H} \mathrm{NMR}\left(400 \mathrm{MHz}, \mathrm{CDCl}_{3}\right) \delta 7.77(\mathrm{~s}, 1 \mathrm{H}), 7.64$ (s, $1 \mathrm{H}), 7.59$ (d, $J=8.3 \mathrm{~Hz}, 1 \mathrm{H}), 7.33$ (dd, $J=5.0,3.0 \mathrm{~Hz}$, 1H), 7.30-7.15 (m, 4H), 7.01 (d, $J=8.3 \mathrm{~Hz}, 1 \mathrm{H}), 5.44$ (s, 2H). ${ }^{13} \mathrm{C} \mathrm{NMR}\left(100 \mathrm{MHz}, \mathrm{CDCl}_{3}\right) \delta 138.2,136.5,133.7$, $133.5,130.2$, 130.1, 128.5, 127.3, 126.7, 126.6, 123.5, 119.5, 118.9, 56.5 .

3-Phenylpyrazolo[5,1-a]isoindole $(38)^{6}$ The reaction of 1 (2-bromobenzyl)-4-phenylpyrazole 36 (0.313 g, $1 \mathrm{mmol}$ ) and PivOK (0.280 g, $2 \mathrm{mmol})$ at $150^{\circ} \mathrm{C}$ during $20 \mathrm{~h}$ in DMA (4 mL) in the presence of $\operatorname{PdCl}\left(\mathrm{C}_{3} \mathrm{H}_{5}\right)(\mathrm{dppb})(12.2$ $\mathrm{mg}, 0.02 \mathrm{mmol})$ under argon affords, after evaporation of the solvent and purification on silica gel, product $\mathbf{3 8}$ in 88\% (0.204 g) yield. ${ }^{1} \mathrm{H}$ NMR (400 MHz, $\left.\mathrm{CDCl}_{3}\right) \delta 7.82$ (d, $J=$ $8.3 \mathrm{~Hz}, 1 \mathrm{H}), 7.77$ (s, 1H), 7.63 (d, $J=8.3 \mathrm{~Hz}, 2 \mathrm{H}), 7.51-$ 7.43 (m, 3H), 7.42-7.30 (m, 3H), 5.17 (s, 2H). ${ }^{13} \mathrm{C}$ NMR $\left(100 \mathrm{MHz}, \mathrm{CDCl}_{3}\right) \delta 142.5,142.1,140.4,133.0,130.9$, 128.7, 128.0, 127.3, 127.2, 126.4, 123.4, 120.2, 115.8, 52.0.

3-(Thiophen-2-yl)-pyrazolo[5,1-a]isoindole (39) The reaction of 1-(2-bromobenzyl)-4-(thiophen-2-yl)-pyrazole 37 (0.319 g, $1 \mathrm{mmol})$ and PivOK (0.280 g, $2 \mathrm{mmol})$ at $150^{\circ} \mathrm{C}$ during $20 \mathrm{~h}$ in DMA (4 mL) in the presence of
$\operatorname{PdCl}\left(\mathrm{C}_{3} \mathrm{H}_{5}\right)(\mathrm{dppb})$ (12.2 mg, $0.02 \mathrm{mmol}$ ) under argon affords, after evaporation of the solvent and purification on silica gel, product 39 in $90 \%\left(0.214\right.$ g) yield. ${ }^{1} \mathrm{H}$ NMR $\left(400 \mathrm{MHz}, \mathrm{CDCl}_{3}\right) \delta 7.80(\mathrm{~d}, J=8.2 \mathrm{~Hz}, 1 \mathrm{H}), 7.74(\mathrm{~s}, 1 \mathrm{H})$, 7.48 (d, $J=8.2 \mathrm{~Hz}, 1 \mathrm{H}), 7.46-7.31$ (m, 5H), 5.15 (s, 2H). ${ }^{13} \mathrm{C}$ NMR $\left(100 \mathrm{MHz}, \mathrm{CDCl}_{3}\right) \delta 142.8,142.5,140.6,133.6$, 131.2 , 128.4, 127.6, 127.5, 126.2, 123.7, 120.5, 120.3, 110.8, 52.3. $\mathrm{C}_{14} \mathrm{H}_{10} \mathrm{~N}_{2} \mathrm{~S}$ (238.31): Calcd C 70.56, H 4.23; Found C 70.54, H 4.50.

\section{5-Chlorodibenzo[c,e]pyrazolo[1,5-a]azepine-4-}

carbonitrile (40) The reaction of 2-(1-(2-bromobenzyl)-4chloropyrazol-5-yl)benzonitrile 10 (0.372 g, $1 \mathrm{mmol})$ and PivOK (0.280 g, $2 \mathrm{mmol})$ at $150^{\circ} \mathrm{C}$ during $20 \mathrm{~h}$ in DMA (4 $\mathrm{mL}$ ) in the presence of $\mathrm{PdCl}\left(\mathrm{C}_{3} \mathrm{H}_{5}\right)(\mathrm{dppb})$ (12.2 $\mathrm{mg}, 0.02$ $\mathrm{mmol}$ ) under argon affords, after evaporation of the solvent and purification on silica gel, product $\mathbf{4 0}$ in 28\% (0.081 g) yield. ${ }^{1} \mathrm{H}$ NMR (400 MHz, $\left.\mathrm{CDCl}_{3}\right) \delta 7.93(\mathrm{~d}, J=8.2 \mathrm{~Hz}$, $1 \mathrm{H}), 7.90$ (d, $J=8.2 \mathrm{~Hz}, 1 \mathrm{H}), 7.68$ (t, $J=7.8 \mathrm{~Hz}, 1 \mathrm{H}), 7.60$ (d, $J=8.2 \mathrm{~Hz}, 1 \mathrm{H}), 7.48-7.40(\mathrm{~m}, 4 \mathrm{H}), 5.30$ (d, $J=14.3$ $\mathrm{Hz}, 1 \mathrm{H}), 4.99$ (d, $J=14.3 \mathrm{~Hz}, 1 \mathrm{H}) .{ }^{13} \mathrm{C}$ NMR (100 MHz, $\left.\mathrm{CDCl}_{3}\right) \delta 140.5,138.3,137.5,137.1,134.9,133.6,133.1$, $130.3,130.0,129.7,129.5,129.0,128.4,118.0,113.8$, 112.6, 55.0. $\mathrm{C}_{17} \mathrm{H}_{10} \mathrm{ClN}_{3}$ (291.73): Calcd C 69.99, H 3.45; Found C 70.17, H 3.57.

\section{5-Bromodibenzo[c,e]pyrazolo[1,5-a]azepine-4-}

carbonitrile (41) The reaction of 2-(4-bromo-1-(2bromobenzyl)-pyrazol-5-yl)benzonitrile 21 (0.417 g, 1 mmol) and PivOK (0.280 g, $2 \mathrm{mmol})$ at $150^{\circ} \mathrm{C}$ during $20 \mathrm{~h}$ in DMA (4 mL) in the presence of $\mathrm{PdCl}\left(\mathrm{C}_{3} \mathrm{H}_{5}\right)(\mathrm{dppb})(12.2$ $\mathrm{mg}, 0.02 \mathrm{mmol})$ under argon affords, after evaporation of the solvent and purification on silica gel, product $\mathbf{4 1}$ in 35\% (0.117 g) yield. ${ }^{1} \mathrm{H}$ NMR (400 MHz, $\left.\mathrm{CDCl}_{3}\right) \delta 7.92$ (d, $J=$ $8.2 \mathrm{~Hz}, 1 \mathrm{H}), 7.90$ (d, $J=8.2 \mathrm{~Hz}, 1 \mathrm{H}), 7.68$ (t, $J=7.8 \mathrm{~Hz}$, $1 \mathrm{H}), 7.61$ (d, $J=8.2 \mathrm{~Hz}, 1 \mathrm{H}), 7.49$ (s, 1H), 7.47-7.40 (m, 3H), 5.32 (d, $J=14.3 \mathrm{~Hz}, 1 \mathrm{H}), 5.01$ (d, $J=14.3 \mathrm{~Hz}, 1 \mathrm{H})$. ${ }^{13} \mathrm{C}$ NMR (100 MHz, $\left.\mathrm{CDCl}_{3}\right) \delta 140.6,140.4,137.4,136.9$, 134.7, 133.5, 130.1, 129.8, 129.6, 129.4, 129.2, 128.3, 118.0, 113.8, 112.6, 97,2, 54.8. $\mathrm{C}_{17} \mathrm{H}_{10} \mathrm{BrN}_{3}$ (336.19): Calcd C 60.73, H 3.00; Found C 60.54, H 2.88.

\section{References}

1. (a) Negishi, E. Ed. Handbook of Organopalladium Chemistry for Organic Synthesis; Wiley-Interscience: New York, 2002; Part III, p 213; (b) Li, J. J.; Gribble, G. W. Palladium in Heterocyclic Chemistry, Pergamon: Amsterdam, 2006; (c) Ackermann, L. Modern Arylation Methods; Wiley: Weinheim, 2009.

2. (a) Akita, Y.; Inoue, A.; Yamamoto, K.; Ohta, A.; Kurihara, T.; Shimizu, M. Heterocycles 1985, 23, 2327-2333.; (b) Ohta, A.; Akita, Y.; Ohkuwa, T.; Chiba, M.; Fukunaga, R.; Miyafuji, A.; Nakata, T.; Tani, N.; Aoyagi, Y. Heterocycles 1990, 31, 1951-1958; (c) Aoyagi, Y.; Inoue, A.; Koizumi, I.; Hashimoto, R.; Miyafuji, A.; Kunoh, J.; Honma, R. Akita, Y.; Ohta, A. Heterocycles 1992, 33, 257-272.

3. For reviews on Pd-catalysed $\mathrm{C}-\mathrm{H}$ functionalisations: (a) Alberico, D.; Scott, M. E.; Lautens, M. Chem. Rev. 2007, 107, 174-238; (b) Satoh, T.; Miura, M. Chem. Lett. 2007, 36, 200-205; (c) Li, B.-J.; Yang, S.-D.; Shi, Z.-J. Synlett 2008, 
949-957; (d) Bellina, F.; Rossi, R. Tetrahedron 2009, 65, 10269-10310; (e) Li, B.-J.; Yang, S.-D.; Shi, Z.-J. Synlett 2008, 949-957; (f) Ackermann, L.; Vincente, R.; Kapdi, A. R. Angew. Chem. Int. Ed. 2009, 48, 9792-9826; (g) Roger, J.; Gottumukkala, A. L.; Doucet, H. ChemCatChem 2010, 2, 2040; (h) Wu, X.-F.; Anbarasan, P.; Neumann, H.; Beller M. Angew. Chem. Int. Ed. 2010, 49, 7316-7319; (i) Sun, C.-L.; Li, B.-J.; Shi, Z.-J. Chem. Commun. 2010, 46, 677-685; (j) Kuhl, N.; Hopkinson, M. N.; Wencel-Delord, J.; Glorius, F. Angew. Chem. Int. Ed. 2012, 51, 10236-10254; (k) Yamaguchi, J.; Yamaguchi, A. D.; Itami, K. Angew. Chem., Int. Ed. 2012, 51, 8960-9009; (l) Wencel-Delord, J.; Glorius, F. Nature Chem. 2013, 5, 369-375; (m) Kuzhushkov, S. I.; Potukuchi, H. K.; Ackermann, L. Catal. Sci. Technol. 2013, 3, 562-571; (n) Rossi, R.; Bellina, F.; Lessi, M.; Manzini, C. Adv. Synth. Catal. 2014, 356, 17-117; (o) He, M.; Soulé, J. F.; Doucet, H. ChemCatChem 2014, 6, 1824-1859; (p) Zhang, M.; Zhang, Y.; Jie, X.; Zhao, H.; Li, G.; Su, W. Org. Chem. Front. 2014, 1, 843-895; (q) Kakiuchi, F.; Kochi, T.; Shinji, M. Synlett 2014, 2390-2414; (r) Liron, F.; Oble, J.; Lorion, M. M.; Poli, G. Eur. J. Org. Chem. 2014, 5863-5883; (s) Yuan, K.; Soulé, J. F.; Doucet, H. ACS Catal. 2015, 5, 978991; (t) Bheeter, C. B.; Chen, L.; Soulé, J.-F.; Doucet, H. Cat. Sci. Technol. 2016, 6, 2005-2049.

4. (a) Goikhman, R.; Jacques, T. L.; Sames, D. J. Am. Chem. Soc. 2009, 131, 3042-3048; (b) Rene, O.; Fagnou, K. Adv. Synth. Catal. 2010, 352, 2116-2120; (c) Gaulier, S. M.; McKay, R.; Swain, N. A. Tetrahedron Lett. 2011, 52, 60006002; (d) Yan, T.; Chen, L.; Bruneau, C.; Dixneuf, P. H.; Doucet, H. J. Org. Chem. 2012, 77, 7659-7664; (e) Bellina, F.; Lessi, M.; Manzini, C. Eur. J. Org. Chem. 2013, 56215630; (f) Kumpulainen, E. T. T.; Pohjakallio, A. Adv. Synth. Catal. 2014, 356, 1555-1561.

5. Iaroshenko, V. O.; Gevorgyan, A.; Davydova, O.; Villinger, A.; Langer, P. J. Org. Chem. 2014, 79, 2906-2915.

6. Choi, Y. L.; Lee, H.; Kim, B. T.; Choi, K.; Heo, J.-N. Adv. Synth. Catal. 2010, 352, 2041-2049.

7. (a) Mateos, C.; Mendiola, J.; Carpintero, M.; Minguez, J. M. Org. Lett. 2010, 12, 4924-4927; (b) Yang, Y.; Kuang, C.; Jin, H.; Yang, Q.; Zhang, Z. Beil. J. Org. Chem. 2011, 16561662; (c) Brahim, M.; Smari, I.; Ben Ammar, H.; Ben Hassine, B.; Soule, J.-F.; Doucet, H. Org. Chem. Front. 2015, 2, 917-926.

8. Smari, I.; Youssef, C.; Yuan, K.; Beladhria, A.; Ben Ammar, H.; Ben Hassine, B.; Doucet, H. Eur. J. Org. Chem. 2014, $1778-1786$.

9. Xu, X.; Zhao, L.; Li, Y.; Soule, J.-F.; Doucet, H. Adv. Synth. Catal. 2015, 357, 2869-2882.

10. (a) Rodriguez-Franco, M. I.; Dorronsoro, I.; HernandezHigueras A. I.; Antequera, G. Tetrahedron Lett. 2001, 42, 863-865; (b) Zhao, Z. G.; Wang, Z. X. Synth. Commun. 2007, 37, 137-147.

11. Cantat, T.; Génin, E.; Giroud, C.; Meyer, G.; Jutand, A. J. Organomet. Chem. 2003, 687, 365-376.

Keywords: palladium, catalysis, $\mathrm{C}-\mathrm{H}$ bond activation, pyrazoles, direct arylation, aryl halides 